\title{
Some new results on modified diagonals
}

\author{
Claire Voisin* \\ CNRS, Institut de Mathématiques de Jussieu and IAS
}

\begin{abstract}
In the paper 12, O'Grady studied $m$-th modified diagonals for a smooth connected projective variety, generalizing the Gross-Schoen modified small diagonal 9]. These cycles $\Gamma^{m}(X, a)$ depend on a choice of reference point $a \in X$ (or more generally a degree 1 zero-cycle). We prove that for any $X, a$, the cycle $\Gamma^{m}(X, a)$ vanishes for large $m$. We also prove the following conjecture of O'Grady: if $X$ is a double cover of $Y$ and $\Gamma^{m}(Y, a)$ vanishes (where $a$ belongs to the branch locus), then $\Gamma^{2 m-1}(X, a)$ vanishes, and we provide a generalization to higher degree finite covers. We finally prove the vanishing $\Gamma^{n+1}\left(X, o_{X}\right)=0$ when $X=S^{[m]}, S$ a $K 3$ surface, and $n=2 m$, which was conjectured by O'Grady and proved by him for $m=2,3$.
\end{abstract}

\section{Introduction}

Let $X$ be a connected smooth projective variety of dimension $n$. We will denote in this paper $\mathrm{CH}_{i}(X)$ the Chow groups of $X$ with rational coefficients and $\mathrm{CH}_{i}(X) /$ alg the groups of $i$-cycles of $X$ with $\mathbb{Q}$-coefficients modulo algebraic equivalence. Let $a \in \mathrm{CH}_{0}(X)$ be a 0 -cycle of degree 1 on $X$. Following Gross-Schoen [9] and O'Grady [12, let us consider for $m \geq 2$ the following $n$-cycle $\Gamma^{m}(X, a)$ in $X^{m}$, which is a modification of the $m$-th small diagonal of $X$ :

$$
\Gamma^{m}(X, a)=\sum_{I \subset\{1, \ldots, m\},|I|=i<m}(-1)^{i} p_{I}^{*}\left(a^{* i}\right) \cdot p_{J}^{*} \Delta_{m-i} \in \mathrm{CH}_{n}\left(X^{m}\right)_{\mathbb{Q}},
$$

where

- $\{1, \ldots, m\}$ is the disjoint union of $I$ and $J$,

- $p_{I}: X^{m} \rightarrow X^{i}$, resp. $p_{J}: X^{m} \rightarrow X^{m-i}$ are the projections onto the products of factors indexed by $I$, resp. $J$,

- $\Delta_{m-i}$ is the small diagonal of $X^{m-i}, \Delta_{1}=X$,

- $a^{* i} \in \mathrm{CH}_{0}\left(X^{i}\right)$ is defined by

$$
a^{* i}=p_{1}^{*} a \cdot \ldots \cdot p_{i}^{*} a .
$$

For example, for $m=2$, we have $\Gamma^{2}(X, a)=\Delta_{X}-a \times X-X \times a$ and $\Gamma^{2}(X, a)=0$ if and only if $X=\mathbb{P}^{1}$ or a point. The modified small diagonal $\Gamma^{3}(X, a)$ appears in several recent works. Gross and Schoen prove that $\Gamma^{3}(X, a)=0$ if $X$ is a hyperelliptic curve and $a$ is a Weierstrass point. This result was greatly extended in [5 by Colombo and van Geemen, who worked with 1-cycles modulo algebraic equivalence and proved that, for a $d$-gonal curve $X$, the cycle $\Gamma^{d+1}(X, a)$ is algebraically equivalent to 0 . Although they do not state their result in this form, but as the vanishing modulo algebraic equivalence of the components $Z_{s}, s \geq d-1$ of the Beauville decomposition (see [1]) of $X$ in its Jacobian, one can show

\footnotetext{
*This research has been supported by The Charles Simonyi Fund and The Fernholz Foundation.
} 
that this is equivalent to the vanishing of $\Gamma^{d+1}(X, a)$ modulo algebraic equivalence. For completeness, we will prove this fact in subsection 4.1

Concerning higher dimensional varieties, Beauville and the author proved in [2] the following theorem:

Theorem 1.1. Let $X$ be a K3 surface. Then there exists a canonical degree 1 zero-cycle $o_{X}$ of $X$ such that

$$
\Gamma^{3}\left(X, o_{X}\right)=0 \text { in } \mathrm{CH}_{2}\left(X^{3}\right)
$$

In fact, $o_{X}$ can be defined as the class in $\mathrm{CH}_{0}(X)$ of any point of $X$ lying on a (singular) rational curve in $X$.

In the paper [12], O'Grady investigates $\Gamma^{m}(X, a)$ for higher $m$. He proves the following results (for $X$ smooth projective connected):

Theorem 1.2. (O'Grady [12]) (i) The cycle $\Gamma^{n+1}(X, a)$ is cohomologous to 0, for $n=$ $\operatorname{dim} X$ and $q(X)=0$. More generally $\Gamma^{m+1}(X, a)$ is cohomologous to 0 if and only if $m \geq \operatorname{dim} X+d$, where $d$ is the dimension of the image of $X$ in its Albanese variety.

(ii) If $\Gamma^{m}(X, a)=0$ then $\Gamma^{m^{\prime}}(X, a)=0$ for $m^{\prime} \geq m$.

(iii) If $p: X \rightarrow Y$ is a ramified double cover and $a$ is a branch point such that $\Gamma^{m}(Y, a)=$ 0 , then for $m=2$ or $m=3, \Gamma^{2 m-1}(X, b)=0$, where $p(b)=a$.

He conjectures that (iii) holds for any $m$ (see [12, Conjecture 5.1]). One of our results is the proof of O'Grady's conjecture, see (i) below, and a generalization to any degree, see (ii) and (iii) below.

Theorem 1.3. Let $p: X \rightarrow Y$ be a degree $d$ finite morphism, where $X$, $Y$ are smooth projective and connected.

(i) Assume $d=2, a \in \mathrm{CH}_{0}(Y)$ is a 0 -cycle of degree 1 supported on the branch locus of $p$, and $b:=\frac{1}{2} p^{*} a \in \mathrm{CH}_{0}(X)$; if $\Gamma^{m}(Y, a)=0$, then $\Gamma^{2 m-1}(X, b)=0$.

(ii) For any $d$, assume $a \in Y$ is a point such that the subscheme $p^{-1}(a)$ is supported on $a$ point $b \in X$. If $\Gamma^{m}(Y, a)=0$, then $\Gamma^{d(m-1)+1}(X, b)=0$.

(iii) For any $d$, let $b:=\frac{1}{d} p^{*} a$ for some 0 -cycle $a \in \mathrm{CH}_{0}(Y)$ of degree 1 . If $\Gamma^{m}(Y, a)=0$ in $\mathrm{CH}_{n}\left(Y^{m}\right) / \mathrm{alg}$, then $\Gamma^{d(m-1)+1}(X, b)=0$ in $\mathrm{CH}_{n}\left(X^{d(m-1)+1}\right) / \mathrm{alg}$.

Statement (i) of Theorem 1.3 has been obtained independently by Moonen and Yin 11.

Remark 1.4. When $Y=\mathbb{P}^{n}$, and $d \leq n+1$, there always exists a point $a \in \mathbb{P}^{n}$ as in (ii) (cf. 8]). In this case, we have $\Gamma^{m}(Y, a)=0$, with $m=n+1$, hence we conclude that for $d$-th covers $X$ of $\mathbb{P}^{n}$ with $d \leq n+1, \Gamma^{d n+1}(X, b)=0$, with $b=\frac{1}{d} p^{*}(p t)$. Note also that any curve $X$ of genus $g$ admits a morphism of degree $d \leq g+1$ to $\mathbb{P}^{1}$, which is totally ramified at one given point $x$. Hence we get $\Gamma^{g+2}(X, x)=0$ for any $x \in X$. This last result is also proved by Moonen and Yin [1] using the Colombo-van Geemen vanishing result.

Remark 1.5. In the case where $Y$ is $\mathbb{P}^{1}$, so $X$ is a $d$-gonal curve, Theorem 1.3 . (iii) gives the vanishing $\Gamma^{d+1}(X, b)=0$ in $\mathrm{CH}_{1}\left(X^{d+1}\right) /$ alg. As explained in Subsection 4.1. this is equivalent to the Colombo-van Geemen theorem [5] mentioned above.

Another application of Theorem 1.3 is the following result, which will be deduced from it in Section 2 using the smash nilpotence result of [14 for cycles algebraically equivalent to 0 :

Corollary 1.6. Let $X$ be a smooth projective(connected) variety of dimension $n$. Then for any $a \in \mathrm{CH}_{0}(X)$ of degree 1 , there exists an integer $m$ such that $\Gamma^{m}(X, a)=0$ in $\mathrm{CH}^{(m-1) n}\left(X^{m}\right)$.

Our second result is the following more precise statement: 
Theorem 1.7. Let $X$ be smooth projective connected of dimension $n$ and let $a \in \mathrm{CH}_{0}(X)$ be of degree 1. Then, if $X$ is swept-out by irreducible curves of genus $g$ supporting a zero-cycle rationally equivalent to $a$, and $m \geq(n+1)(g+1)$, one has $\Gamma^{m}(X, a)=0$ in $\mathrm{CH}^{(m-1) n}\left(X^{m}\right)$.

Note that such a $g$ always exists: Indeed, consider curves in $X$ which are complete intersections of ample hypersurfaces containing the support of the cycle $a$. For sufficiently high degree such hypersurfaces, these curves will sweeep-out $X$, and thus we can take for $g$ the genus of the generic such curves. In the case where $X$ is a $K 3$ surface, we know that $X$ is swept-out by elliptic curves supporting the canonical 0-cycle. Hence we get from Theorem 1.7 the vanishing $\Gamma^{6}\left(X, o_{X}\right)=0$, which is not optimal in view of the relation (3) in Theorem 1.1.

We finally turn to the case of hyper-Kähler manifolds. For $K 3$ surfaces, one can get as a consequence of (3) the following properties of $o_{X}$ (note however that property 1 below is used to prove (3) so that we do not actually recover it from (3). Nevertheless, the consequences 1 and 2 indicate that surfaces satisfying (3) are quite special):

1. The intersection of two divisors $D, D^{\prime}$ on $X$ is proportional to $o_{X}$ in $\mathrm{CH}_{0}(X)$.

2. The second Chern class $c_{2}(X)$ is equal to $24 o_{X}$.

In the paper [12], O'Grady formulates the following generalization of (3):

Conjecture 1.8. (O'Grady, [12, Conjecture 0.1]) Let $X$ be a hyper-Kähler n-fold. Then there exists a canonical 0 -cycle $o_{X} \in \mathrm{CH}_{0}(X)$ of degree 1 such that $\Gamma^{n+1}\left(X, o_{X}\right)=0$ in $\mathrm{CH}_{n}\left(X^{n+1}\right)$.

Note that by Theorem 1.2. (i), we have $\left[\Gamma^{n+1}\left(X, o_{X}\right)\right]=0$ in $H^{*}\left(X^{n+1}, \mathbb{Q}\right)$ and that this is optimal. Conjecture 1.8 thus states that the cycles $\Gamma^{k}\left(X, o_{X}\right)$ vanish in $\mathrm{CH}\left(X^{k}\right)$ once they vanish in $H^{*}\left(X^{k}, \mathbb{Q}\right)$, which is very different from the situation encountered in the case of curves (except for the hyperelliptic ones).

O'Grady establishes this conjecture for the punctual Hilbert schemes $S^{[2]}$ and $S^{[3]}$ of a $K 3$ surface. The canonical 0 -cycle $o_{X}$, for $X=S^{[n]}$, is naturally defined as the class in $\mathrm{CH}_{0}(X)$ of any point of $X$ lying over $n o_{S} \in S^{(n)}$, for some representative $o_{S} \in S$ of the canonical 0-cycle of $S$. We prove in section 5 Conjecture 1.8 for punctual Hilbert schemes $X=S^{[n]}$ of $K 3$ surfaces, and for any $n$, using methods from [16] and recent results of Yin [20]:

Theorem 1.9. Let $S$ be a $K 3$ surface, and let $X=S^{[m]}$. Then

$$
\Gamma^{n+1}\left(X, o_{X}\right)=0 \text { in } \mathrm{CH}_{n}\left(X^{n+1}\right) .
$$

where $o_{X}$ is the canonical 0 -cycle on $X$ coming from the canonical 0 -cycle of $S$, and $n=$ $\operatorname{dim} X=2 m$.

Note that one can recover from (41) the following result, which had been in fact already proved in [16, Theorem 1.5].

Corollary 1.10. The intersection of $n$ divisors on $X$ is proportional to o $o_{X}$ in $\mathrm{CH}_{0}(X)$.

For the proof of Theorem 1.9, we will need three tools. The first ingredient is similar to what we did in [16, namely we will use the de Cataldo-Migliorini theorem [4] and will prove Proposition 5.6 in order to reduce to computations in the Chow rings of the self-products $S^{k}$. The second ingredient is very new and it is provided by Yin's recent result [20] saying that the cohomological relations between the big diagonals of a regular surface and the pull-back of the class of a point are generated (modulo trivial relations) by the pull-backs of the Kimura relation and the cohomological counterpart $\left[\Gamma^{3}\left(S, o_{S}\right)\right]=0$ in $H^{8}\left(S^{3}, \mathbb{Q}\right)$ of the relation (3) (see also [12, Proposition 1.3]). We then argue that the Kimura relation is not needed in our context, while the relation $\Gamma^{3}\left(S, o_{S}\right)=0$ is satisfied in the Chow ring by Theorem 1.1 . 
To conclude, let us remark that the following conjecture in the same spirit as Conjecture 1.8 was stated first in [16] for $K 3$ surfaces, and then in [13] for general hyper-Kähler manifolds:

Conjecture 1.11. Let $X$ be a projective hyper-Kähler manifold and $n>0$ be an integer. Then there exists a canonical 0 -cycle $o_{X} \in \mathrm{CH}_{0}(X)$ such that any polynomial relation between the cohomology classes $\operatorname{pr}_{i}^{*}\left[o_{X}\right], i \leq n, \operatorname{pr}_{i j}^{*}\left[\Delta_{X}\right], i \neq j \leq n$, already holds in $\mathrm{CH}\left(X^{n}\right)$.

O'Grady's conjecture 1.8 is the particular case of Conjecture 1.11 which concerns the class $\Gamma^{n+1}\left(X, o_{X}\right), n=\operatorname{dim} X$. As explained in [18] in the case of $K 3$ surfaces, Conjecture 1.11 is extremely strong since it implies finite dimensionality in the Kimura sense, with very important consequences established by Kimura [10, in particular on the nilpotency of self-correspondences homologous to 0. O'Grady's conjecture 1.8 does not seem to have such implications, so it is possibly of a nature different from Conjecture 1.11.

The paper is organized as follows: in section 2 we introduce variants $\Gamma^{1, m}(X, a)$ of the cycles $\Gamma^{m}(X, a)$ which lie in $\mathrm{CH}_{n}\left(X^{m+1}\right), n=\operatorname{dim} X$ and relate them to $\Gamma^{m}(X, a)$. In section 3, we will prove Theorem 1.7. Theorem 1.3 will be proved in Section 4 and Theorem [1.9 will be proved in section 5. The last subsection 5.2 is devoted to the sketch of the proof of a general theorem (Theorem 5.12) concerning universally defined cycles on quasiprojective surfaces, which is used in the proof of Theorem 1.9. This result is of independent interest and its complete proof will be given together with further applications in [19].

Thanks. This paper has been completed at the Institute for Advanced Study, which I thank for ideal working conditions. I also thank Lie Fu, Ben Moonen, Kieran O'Grady, Burt Totaro and Qizheng Yin for interesting discussions related to this work, and the referee for his/her useful suggestions and comments.

\section{Cycles $\Gamma^{1, m}(X, a)$}

We first introduce the following notation: $X$ being smooth projective, and $a \in \mathrm{CH}_{0}(X)_{\mathbb{Q}}$ being a zero-cycle of degree 1 , we define $\Gamma^{1, m}(X, a) \in \mathrm{CH}_{n}\left(X^{m+1}\right)_{\mathbb{Q}}$ by

$$
\Gamma^{1, m}(X, a):=\prod_{1 \leq i \leq m}\left(p_{0 i}{ }^{*} \Delta_{X}-p_{i}^{*} a\right)
$$

where

1. $\Delta_{X} \subset X \times X$ is the diagonal of $X$,

2. $p_{0 i}: X^{m+1} \rightarrow X \times X$ is the projection on the product of the first and $i+1$-th factors,

3. $p_{i}: X^{m+1} \rightarrow X$ is the projection on the $i+1$-th factor (our factors are indexed by $\{0, \ldots, m\})$.

The cycles $\Gamma^{m}(X, a)$ and $\Gamma^{1, m}(X, a)$ are related as follows:

Lemma 2.1. We have the following formula:

$$
\Gamma^{m}(X, a)=p_{1, \ldots, m *}^{\prime} \Gamma^{1, m}(X, a),
$$

where we index the factors of $X^{m+1}$ by $\{0, \ldots, m\}$ and $p_{1, \ldots, m}^{\prime}$ is the projection from $X^{m+1}$ to the products $X^{m}$ of its last $m$ factors. We also have:

$$
\Gamma^{m+1}(X, a)=\Gamma^{1, m}(X, a)-p_{0}^{*} a \cdot p_{1, \ldots, m}^{\prime}{ }^{*}\left(\Gamma^{m}(X, a)\right) .
$$


Proof. This is almost immediate. Developing the product in (5), we get

$$
\Gamma^{1, m}(X, a)=\sum_{I \subset\{1, \ldots, m\},|I|=i}(-1)^{i} p_{I}^{\prime *}\left(a^{* i}\right) \cdot p_{0, J}^{*} \Delta_{m+1-i},
$$

where $I \bigsqcup J=\{1, \ldots, m\}, p_{0, J}$ is the projection from $X^{m+1}$ to the product $X^{m+1-i}$ of factors indexed by $\{0\} \cup J$ and $p_{I}^{\prime}$ is the projection from $X^{m+1}$ to the product $X^{i}$ of the factors indexed by $I \subset\{1, \ldots, m\}$. Applying $p_{1, \ldots, m *}^{\prime}: \mathrm{CH}_{n}\left(X^{m+1}\right)_{\mathbb{Q}} \rightarrow \mathrm{CH}_{n}\left(X^{m}\right)_{\mathbb{Q}}$, we get by the projection formula, using the fact that $p_{I}^{\prime}=p_{I} \circ p_{1, \ldots, m}^{\prime}$ :

$$
p_{1, \ldots, m *}^{\prime} \Gamma^{1, m}(X, a)=\sum_{I \subset\{1, \ldots, m\},|I|=i}(-1)^{i} p_{I}^{*}\left(a^{* i}\right) \cdot p_{1, \ldots, m *}^{\prime}\left(p_{0, J}^{*} \Delta_{m+1-i}\right) .
$$

Formula (6) then follows from the fact that $p_{J}^{*} \Delta_{m-i}=p_{1, \ldots, m *}^{\prime}\left(p_{0, J}^{*} \Delta_{m+1-i}\right)$ in $\mathrm{CH}_{n}\left(X^{m}\right)$.

As for (7), we first write formula (1) for $X^{m+1}$, where as above we index the factors of $X^{m+1}$ by $\{0, \ldots, m\}$. This gives us

$$
\Gamma^{m+1}(X, a)=\sum_{I \subset\{0, \ldots, m\}, i=|I| \leq m}(-1)^{i} p_{I}^{\prime *}\left(a^{* i}\right) \cdot p_{J}^{\prime *} \Delta_{m+1-i} \in \mathrm{CH}_{n}\left(X^{m+1}\right)_{\mathbb{Q}} .
$$

We now separate the terms where $0 \notin I$, which by (8) exactly give $\Gamma^{1, m}(X, a)$, and the terms where $0 \in I$, which exactly give $-p_{0}^{*} a \cdot p_{1, \ldots, m}^{\prime}{ }^{*}\left(\Gamma^{m}(X, a)\right)$.

We deduce the following

Proposition 2.2. The vanishing of $\Gamma^{m}(X, a)$ in $\mathrm{CH}_{n}\left(X^{m}\right)$ is equivalent to the vanishing of $\Gamma^{1, m}(X, a)$ in $\mathrm{CH}_{n}\left(X^{m+1}\right)$.

Proof. Indeed, if $\Gamma^{1, m}(X, a)=0$ then $\Gamma^{m}(X, a)=0$ by (6). Conversely, if $\Gamma^{m}(X, a)=0$, then [12, Proposition 2.4] shows that also $\Gamma^{m+1}(X, a)=0$. Formula (7) then implies that $\Gamma^{1, m}(X, a)=0$.

A consequence of this result is the following statement comparing $\Gamma^{m}(X, a)$ and $\Gamma^{m}(X, b)$, for two 0-cycles $a, b \in \mathrm{CH}_{0}(X)$ of degree 1 .

Corollary 2.3. If $\Gamma^{m}(X, a)=0$ and the cycle $b-a$ satisfies $(b-a)^{* k}=0$ in $\mathrm{CH}_{0}\left(X^{k}\right)$, then $\Gamma^{m+k}(X, b)=0$.

Here we refer to (2) for the definition of the $*$-product (or external product) of cycles.

Proof. Indeed, by Proposition 2.2, the assumption is equivalent to the vanishing conditions:

$$
\begin{gathered}
\Gamma^{1, m}(X, a)=\prod_{i=1}^{i=m}\left(p_{0 i}^{*}\left(\Delta_{X}\right)-p_{i}^{*} a\right)=0 \text { in } \mathrm{CH}_{n}\left(X^{m+1}\right), n=\operatorname{dim} X \\
\prod_{i=1}^{i=k} p_{i}^{*}(b-a)=0 \text { in } \mathrm{CH}_{0}\left(X^{k}\right) .
\end{gathered}
$$

On the other hand, the conclusion is equivalent to the vanishing

$$
\Gamma^{1, m+k}(X, a)=\prod_{i=1}^{m+k}\left(p_{0 i}^{*}\left(\Delta_{X}\right)-p_{i}^{*} b\right)=0 \text { in } \mathrm{CH}_{n}\left(X^{m+k+1}\right) .
$$

We now write $b=a+(b-a)$, getting

$$
\Gamma^{1, m+k}(X, b)=\prod_{i=1}^{m+k}\left(\left(p_{0 i}^{*}\left(\Delta_{X}\right)-p_{i}^{*} a\right)-p_{i}^{*}(b-a)\right)
$$

and develop the product. In the developed expression, the product of $\geq m$ terms of the form $p_{0 i}^{*}\left(\Delta_{X}\right)-p_{i}^{*} a$ is 0 and the product of $\geq k$ terms of the form $p_{i}^{*}(b-a)$ is 0 . Hence we conclude that each monomial in the development is 0 . 
Here is another corollary of Proposition 2.2. It shows how to deduce Corollary 1.6 from Theorem 1.3, and thus gives another proof of the nilpotency statement of Theorem 1.7 with no estimate on the nilpotency index.

Corollary 2.4. Let $X$ be a smooth projective connected variety and let a be a 0-cycle of degree 1 on $X$ such that $\Gamma^{m}(X, a)=0$ in $\mathrm{CH}\left(X^{m}\right) /$ alg. Then for any 0 -cycle $b$ of degree 1 on $X$, there is an integer $M$ such that $\Gamma^{M}(X, b)=0$ in $\mathrm{CH}\left(X^{M}\right)$.

Proof. As $a$ and $b$ are algebraically equivalent, we also have $\Gamma^{m}(X, b)=0$ in $\mathrm{CH}\left(X^{m}\right) /$ alg. By Proposition 2.2 which is true and proved in the same way for cycles modulo algebraic equivalence (observing that [12, Proposition 2.4] is true as well for cycles modulo algebraic equivalence), this is equivalent to the fact that $\Gamma^{1, m}(X, b)$ is algebraically equivalent to 0 in $X^{m+1}$. By the smash-nilpotence result of Voevodsky [14, there is an integer $N$ such that the cycle $\Gamma^{1, m}(X, b)^{* N}$ vanishes identically in $\mathrm{CH}\left(X^{N(m+1)}\right)$. Thus its restriction to $X^{N m+1}$ embedded in $X^{N(m+1)}$ by the small diagonal on the factors of index $0, m+1,2 m+$ $1, \ldots,(N-1) m+1$ also vanishes in $\mathrm{CH}\left(X^{N m+1}\right)$. But this restricted cycle is nothing but $\Gamma^{1, N m}(X, b)$.

The following criterion for the vanishing of $\Gamma^{m}(X, a)$ will be used in Section 4 Here we consider more generally the vanishing of $\Gamma^{m}(X, a)$ modulo an adequate equivalence relation $R$ which in applications will be rational or algebraic equivalence. We need an assumption on the 0 -cycle $a$ of degree 1 , namely

$$
p_{1}^{*} a \cdot p_{2}^{*} a=\Delta_{*} a \text { in } \mathrm{CH}_{0}(X \times X) / R,
$$

where $\Delta$ is the diagonal inclusion map of $X$ in $X \times X$. This assumption is satisfied for any $R$ if $a$ is a point, or for any 0 -cycle if $R$ is algebraic equivalence, and $X$ is connected.

Proposition 2.5. Assume a satisfies (10). Then $\Gamma^{m}(X, a)=0$ in $\mathrm{CH}\left(X^{m}\right) / R$ if and only if

$$
\Gamma^{1, m-1}(X, a)=p_{0}^{*} a \cdot \Gamma \text { in } \mathrm{CH}_{n}\left(X^{m}\right) / R, n=\operatorname{dim} X
$$

for some cycle $\Gamma \in \mathrm{CH}_{2 n}\left(X^{m}\right) / R$.

The proof of Proposition 2.5 will use the following

Lemma 2.6. Assume the degree 1 zero-cycle a of $X$ satisfies (10). Then for any $Y$ and any cycle $\Gamma \in \mathrm{CH}(X \times Y) / R$, the following formula holds:

$$
p_{X}^{*} a \cdot \Gamma=p_{X}^{*} a \cdot p_{Y}^{*} \Gamma_{a} \text { in } \mathrm{CH}(X \times Y) / R,
$$

where

$$
\Gamma_{a}:=p_{Y *}\left(p_{X}^{*} a \cdot \Gamma\right) \in \mathrm{CH}(Y) / R .
$$

Proof. Let $a=\sum_{i} n_{i} a_{i}$, where $a_{i} \in X$. Then

$$
p_{X}^{*} a \cdot \Gamma=\sum_{i} n_{i} p_{X}^{*} a_{i} \cdot \Gamma=\sum_{i} n_{i} a_{i} \times \Gamma_{a_{i}}=\sum_{i} n_{i} p_{X}^{*} a_{i} \cdot p_{Y}^{*} \Gamma_{a_{i}},
$$

where $\Gamma_{a_{i}} \in \mathrm{CH}(Y) / R$ is the restriction of $\Gamma$ to $a_{i} \times Y$. So we need to prove that, assuming (10),

$$
\sum_{i} n_{i} p_{X}^{*} a_{i} \cdot p_{Y}^{*} \Gamma_{a_{i}}=p_{X}^{*} a \cdot p_{Y}^{*} \Gamma_{a} \text { in } \mathrm{CH}(X \times Y) / R
$$

where $\Gamma_{a}=\sum_{i} n_{i} \Gamma_{a_{i}} \in \mathrm{CH}(Y) / R$. Note that (10) is exactly the case of (11) where $X=Y$ and $\Gamma$ is the diagonal of $X$. The general case is then deduced from this one by introducing $X \times X \times Y$ with its various projections to $X, Y$ and $X \times Y$. Namely, let 
- $p_{i, X, Y}: X \times X \times Y \rightarrow X \times Y, i=1,2$, be the projections onto the product of the first and the third (resp. the second and the third) factor,

- $p_{i, X}: X \times X \times Y \rightarrow X, i=1,2$ be the projection on the first, resp. second, factor and

- $p_{X, X}: X \times X \times Y \rightarrow X \times Y \rightarrow X \times X$ be the projection onto the product of the first two factors.

The left hand side of (11) is clearly equal to

$$
p_{2, X, Y *}\left(p_{1, X}^{*} a \cdot p_{X, X}^{*} \Delta_{X} \cdot p_{2, X, Y}^{*} \Gamma\right) .
$$

Formula (10) tells us that on $X \times X, p_{1}^{*} a \cdot \Delta_{X}=p_{1}^{*} a \cdot p_{2}^{*} a$ modulo $R$, so that

$p_{2, X, Y *}\left(p_{1, X}^{*} a \cdot p_{X, X}^{*} \Delta_{X} \cdot p_{2, X, Y}^{*} \Gamma\right)=p_{2, X, Y *}\left(p_{1, X}^{*} a \cdot p_{2, X}^{*} a \cdot p_{2, X, Y}^{*} \Gamma\right)$ in $\mathrm{CH}(X \times Y) / R$

As $a$ has degree 1 , the right hand side of (12) is equal by the projection formula to

$$
p_{X}^{*} a \cdot p_{Y}^{*} \Gamma_{a},
$$

proving (11).

Proof of Proposition 2.5. We have by (7)

$$
\Gamma^{m}(X, a)=\Gamma^{1, m-1}(X, a)-p_{0}^{*} a \cdot p_{1, \ldots, m-1}^{*} \Gamma^{m-1}(X, a)
$$

so if $\Gamma^{m}(X, a)=0$ in $\mathrm{CH}\left(X^{m}\right) / R$, we get

$$
\Gamma^{1, m-1}(X, a)=p_{0}^{*} a \cdot p_{1, \ldots, m-1}^{*} \Gamma^{m-1}(X, a) \text { in } \mathrm{CH}\left(X^{m}\right) / R \text {. }
$$

This proves one direction (for which we do not need (10)). In the other direction, we assume (10) and

$$
\Gamma^{1, m-1}(X, a)=p_{0}^{*} a \cdot \Gamma \text { in } \mathrm{CH}_{n}\left(X^{m}\right) / R
$$

for some cycle $\Gamma \in \mathrm{CH}_{2 n}\left(X^{m}\right) / R$. We now use Lemma 2.6 which gives

$$
p_{0}^{*} a \cdot \Gamma=p_{0}^{*} a \cdot p_{1, \ldots, m-1}^{*}\left(p_{1, \ldots, m-1 *}\left(p_{0}^{*} a \cdot \Gamma\right)\right) .
$$

By (13), this gives

$$
p_{0}^{*} a \cdot \Gamma=p_{0}^{*} a \cdot p_{1, \ldots, m-1}^{*}\left(p_{1, \ldots, m-1 *}\left(\Gamma^{1, m-1}(X, a)\right)\right) \text { in } \mathrm{CH}_{n}\left(X^{m}\right) / R .
$$

As $p_{1, \ldots, m-1 *}\left(\Gamma^{1, m-1}(X, a)\right)=\Gamma^{m-1}(X, a)$ by (6) , we get

$$
\Gamma^{1, m-1}(X, a)=p_{0}^{*} a \cdot p_{1, \ldots, m-1}^{*}\left(\Gamma^{m-1}(X, a)\right) \text { in } \mathrm{CH}_{n}\left(X^{m}\right) / R .
$$

Using (7), we conclude that

$$
\Gamma^{m}(X, a)=\Gamma^{1, m-1}(X, a)-p_{0}^{*} a \cdot p_{1, \ldots, m-1}^{*}\left(\Gamma^{m-1}(X, a)\right)=0 \text { in } \mathrm{CH}_{n}\left(X^{m}\right) / R .
$$




\section{Proof of Theorem 1.7}

We prove in this section Theorem 1.7, that is the following statement:

Theorem 3.1. Let $X$ be a variety of dimension $n$ and let $a \in \mathrm{CH}_{0}(X)$ be of degree 1 . If $X$ is swept-out by irreducible curves of genus $\leq g$ supporting a 0 -cycle rationally equivalent to $a$, and $m \geq(n+1)(g+1)$, then $\Gamma^{m}(X, a)=0$.

Note that for $g=0$, we get the following corollary:

Corollary 3.2. Let $X$ be a rationally connected manifold of dimension $n$. Then $\Gamma^{n+1}(X, o)=$ 0 for any point $o \in X$.

This corollary will be improved at the end of this section in Theorem 3.6

Proof of Theorem 3.1. By Proposition 2.2, it suffices to prove the vanishing of $\Gamma^{1, m}(X, a)$. Let us see $\Gamma^{1, m}(X, a)$ as a correspondence between $X$ and $X^{m}$. Then for any $x \in X$, we have

$$
\Gamma^{1, m}(X, a)_{\mid x \times X^{m}}=(x-a)^{* m} \text { in } \mathrm{CH}_{0}\left(X^{m}\right)_{\mathbb{Q}} .
$$

Recall now the following result proved in [14, [15]:

Lemma 3.3. Let $C$ be a smooth connected curve of genus $g$, and let $z \in \mathrm{CH}_{0}(C)_{\mathbb{Q}}$ be a 0 -cycle of degree 0 on $C$. Then for $k>g, z^{* k}=0$ in $\mathrm{CH}_{0}\left(C^{k}\right)_{\mathbb{Q}}$.

Our assumption is now that $X$ is swept out by irreducible curves of genus $\leq g$ supporting a 0-cycle rationally equivalent to $a$. This means that for any $x \in X$, there is a smooth connected curve $C_{x}$ of genus $\leq g$ mapping to $X$ via a morphism $f_{x}$, a point $x^{\prime} \in C_{x}$ such that $f_{x}\left(x^{\prime}\right)=x$ and a 0 -cycle $a^{\prime} \in \mathrm{CH}_{0}\left(C_{x}\right)_{\mathbb{Q}}$ of degree 1 , such that $f_{x *}\left(a^{\prime}\right)=a$ in $\mathrm{CH}_{0}(X)_{\mathbb{Q}}$. It is then clear that

$$
f_{x *}^{k}\left(\left(x^{\prime}-a^{\prime}\right)^{* k}\right)=(x-a)^{* k} \text { in } \mathrm{CH}_{0}\left(X^{k}\right)_{\mathbb{Q}} .
$$

We thus conclude by Lemma 3.3 that for $k>g$, and for any $x \in X$

$$
\Gamma^{1, k}(X, a)_{\mid x \times X^{k}}=(x-a)^{* k}=0 \text { in } \mathrm{CH}_{0}\left(X^{k}\right)_{\mathbb{Q}} .
$$

We use now the following general principle which is behind the Bloch-Srinivas decomposition of the diagonal [3], see [18, 3.1]:

Theorem 3.4. Let $\phi: W \rightarrow Y$ be a morphism, where $W$ is smooth of dimension $m$. Let $Z$ be a codimension $k$ cycle on $W$. Assume that, for general $y \in Y$, the restriction $Z_{\mid W_{y}}$ vanishes in $\mathrm{CH}^{k}\left(W_{y}\right)$. Then there is a dense Zariski open set $U \subset Y$, such that $Z_{U}=0$ in $\mathrm{CH}^{k}\left(W_{U}\right)$. Equivalently, there exist a nowhere dense closed algebraic subset $D \varsubsetneqq Y$ and a cycle $Z^{\prime} \in \mathrm{CH}_{m-k}\left(W_{D}\right)_{\mathbb{Q}}$ such that

$$
Z=Z^{\prime} \text { in } \mathrm{CH}^{k}(W)_{\mathbb{Q}} .
$$

(Here we use the notation $W_{D}:=\phi^{-1}(D), W_{U}:=\phi^{-1}(U)$.) Applying this statement to $Y=X, W=X^{k+1}, \phi$ the projection to the first factor and $Z=\Gamma^{1, k}(X, a)$, we conclude from (14) that under the assumptions of Theorem 3.1. there exists for $k>g$ a proper closed algebraic subset $D \varsubsetneqq X$, such that $\Gamma^{1, k}(X, a)$ is rationally equivalent to a cycle supported on $D \times X^{k}$.

Recall now the formula (5) defining $\Gamma^{1, k}$ :

$$
\Gamma^{1, k}(X, a):=\prod_{1 \leq i \leq k}\left(p_{0 i}^{*} \Delta_{X}-p_{i}^{*} a\right) .
$$

It follows immediately that

$$
\Gamma^{1, k+k^{\prime}}(X, a)=p_{0,1 \leq i \leq k}^{*} \Gamma^{1, k}(X, a) \cdot p_{0, k+1 \leq i \leq k+k^{\prime}}^{*} \Gamma^{1, k^{\prime}}(X, a),
$$


where

$$
p_{0,1 \leq i \leq k}: X^{k+k^{\prime}+1} \rightarrow X^{k+1}
$$

is the projection on the product of the $k+1$ first factors and

$$
p_{0, k+1 \leq i \leq k+k^{\prime}}: X^{k+k^{\prime}+1} \rightarrow X^{k^{\prime}+1}
$$

is the projection on the product of the first factor (indexed by 0 ) and the last $k^{\prime}$ factors.

For $m \geq(n+1)(g+1)$, we write $m=(n+1)(g+1)+r$, for some $r \geq 0$ and we get from (15):

$$
\begin{gathered}
\Gamma^{1, m}(X, a)=p_{0,1 \leq i \leq g+1}^{*}\left(\Gamma^{1, g+1}\right) \cdot p_{0, g+2 \leq i \leq 2(g+1)}^{*}\left(\Gamma^{1, g+1}\right) \\
\ldots p_{0, n(g+1)+1 \leq i \leq(n+1)(g+1)}^{*}\left(\Gamma^{1, g+1}\right) \cdot p_{0,(n+1)(g+1)+1 \leq i \leq(n+1)(g+1)+r}^{*}\left(\Gamma^{1, r}\right) .
\end{gathered}
$$

Now we proved that the cycle $\Gamma^{1, g+1}$ is supported (via the first projection $X^{g+2} \rightarrow X$ ) over a proper algebraic subset $D \varsubsetneqq X$, and by the easy moving Lemma 3.5 below, we can choose closed algebraic subsets $D_{1}, \ldots, D_{n+1}$ such that $\cap_{i} D_{i}=\emptyset$ and $\Gamma^{1, g+1}$ is supported (via the first projection $X^{g+2} \rightarrow X$ ) over the proper algebraic subset $D_{i} \varsubsetneqq X$ for each $i$.

Then we conclude that for $m \geq(n+1)(g+1), \Gamma^{1, m}(X, a)$ is supported (via the first projection $\left.X^{(n+1)(g+1)+r+1} \rightarrow X\right)$ over the proper algebraic subset $\cap_{i} D_{i}=\emptyset$, and thus is equal to 0 .

Lemma 3.5. Let $Y$ be irreducible and let $Z$ be a cycle on a product $Y \times W$. Assume there exists a proper closed algebraic subset $D \varsubsetneqq Y$ such that $Z$ is rationally equivalent to a cycle $Z^{\prime}$ supported on $D \times W$. Then for any finite set of points $y_{1}, \ldots, y_{l} \in Y$, there is a $D^{\prime} \varsubsetneqq Y$ such that none of the $y_{j}$ 's belongs to $D^{\prime}$ and $Z$ is rationally equivalent to a cycle $Z^{\prime \prime}$ supported on $D^{\prime} \times W$.

Proof. Let $\tau: \widetilde{D} \rightarrow D$ be a desingularization of $D \stackrel{i}{\hookrightarrow} Y$. The cycle $Z^{\prime}$ of $D \times W$ with rational coefficients lifts to a cycle $\widetilde{Z}^{\prime}$ of $\widetilde{D} \times W$. Let $\tilde{i}=i \circ \tau: \widetilde{D} \rightarrow Y$ be the natural map and let $\Gamma_{\tilde{i}} \subset \widetilde{D} \times Y$ be its graph. Since $\Gamma_{\tilde{i}}$ has codimension $n=\operatorname{dim} Y$, and dimension $\leq n-1$, there is a cycle $\Gamma^{\prime} \subset \widetilde{D} \times Y$ rationally equivalent to $\Gamma_{\tilde{i}}$ and not intersecting $\widetilde{D} \times\left\{y_{1}, \ldots, y_{l}\right\}$. In other words, $\operatorname{pr}_{2}\left(\operatorname{Supp} \Gamma^{\prime}\right)$ does not contain any of the points $y_{i}$. We have by assumption

$$
\begin{aligned}
Z=\left(i, I d_{W}\right)_{*} Z^{\prime} & =\left(\tilde{i}, I d_{W}\right)_{*} \widetilde{Z}^{\prime}=\left(\Gamma_{\tilde{i}}, I d_{W}\right)_{*}\left(\widetilde{Z}^{\prime}\right) \\
& =\left(\Gamma^{\prime}, I d_{W}\right)_{*}\left(\widetilde{Z}^{\prime}\right)
\end{aligned}
$$

in $\mathrm{CH}(Y \times W)$. Now, the cycle $\left(\Gamma^{\prime}, I d_{W}\right)_{*}\left(\widetilde{Z}^{\prime}\right)$ is supported on $p r_{2}\left(\operatorname{Supp} \Gamma^{\prime}\right) \times W$, so the result is proved with $D^{\prime}=p r_{2}\left(\operatorname{Supp} \Gamma^{\prime}\right)$, and $Z^{\prime \prime}=\left(\Gamma^{\prime}, I d_{W}\right)_{*}\left(\widetilde{Z}^{\prime}\right)$.

To conclude this section, let us observe that the same scheme of proof proof applies to give the following result, which is a generalization of Corollary 3.2.

Theorem 3.6. Let $X$ be a connected smooth projective variety with $\mathrm{CH}_{0}(X)=\mathbb{Z}$. Then for the canonical degree 10 -cycle o on $X, \Gamma^{n+1}(X, o)=0$ in $\mathrm{CH}_{n}\left(X^{n+1}\right)$, where $n=\operatorname{dim} X$.

Proof. Indeed, the Bloch-Srinivas decomposition of the diagonal [3] gives an equality

$$
\Delta_{X}-X \times o=Z \text { in } \mathrm{CH}_{n}(X \times X),
$$

where $Z$ is supported over $D \times X$, for some divisor $D \subset X$. By Lemma 3.5, we can write such a decomposition with $n+1$ divisors $D_{1}, \ldots, D_{n+1}$ such that $\cap_{i} D_{i}=\emptyset$. We then conclude that $\Gamma^{1, n+1}(X, o)=\prod_{i=1}^{n+1} p_{0 i}^{*}\left(\Delta_{X}-X \times o\right)$ is equal to 0 in $\mathrm{CH}_{n}\left(X^{n+2}\right)$, and it follows from Proposition 2.2 that $\Gamma^{n+1}(X, o)=0$ in $\mathrm{CH}_{n}\left(X^{n+1}\right)$. 


\section{Proof of Theorem 1.3}

We will first give the proof of Theorem 1.3. (i). Let us recall the statement:

Theorem 4.1. Let $Y$ be smooth projective, and let $\pi: X \rightarrow Y$ be a degree 2 finite morphism, where $X$ is smooth projective. Let $a \in \mathrm{CH}_{0}(Y)$ be a 0 -cycle of degree 1 supported on the branch locus of $\pi$. Then if $\Gamma^{m}(Y, a)=0$, we have $\Gamma^{2 m-1}(X, b)=0$, where $b=\frac{1}{2} \pi^{*} a \in$ $\mathrm{CH}_{0}(X)$.

Remark 4.2. The assumption made on $a$ and $b$ is maybe not optimal, but in any case the condition $b=\frac{1}{2} \pi^{*} a$ is not sufficient. Indeed, consider the case where $Y$ is connected with $\Gamma^{m}(Y, a)=0$, and $X$ consists of two copies of $Y$ with $b=\frac{1}{2} \pi^{*} a \in \mathrm{CH}_{0}(X)$. Then $\Gamma^{k}(X, b)$ is different from 0 for any $k$ (in fact it is not even cohomologous to 0 ).

We will denote by $\pi_{2}=(\pi, \pi): X \times X \rightarrow Y \times Y$. Let $i: X \rightarrow X$ be the involution of $X$ over $Y$ and $\Gamma_{i} \subset X \times X$ be its graph. We then have

$$
\pi_{2}^{*}\left(\Delta_{Y}\right)=\Delta_{X}+\Gamma_{i}
$$

Let

$$
\Delta_{X}^{+}=\pi_{2}^{*}\left(\Delta_{Y}\right)=\Delta_{X}+\Gamma_{i}, \Delta_{X}^{-}=\Delta_{X}-\Gamma_{i}
$$

We thus have

$$
2 \Delta_{X}=\Delta_{X}^{+}+\Delta_{X}^{-}
$$

Lemma 4.3. Under the assumptions of Theorem 4.1, we have the following equalities in $\mathrm{CH}_{n}(X \times X \times X), n:=\operatorname{dim} X$.

$$
\begin{aligned}
p_{12}^{*} \Delta_{X}^{-} \cdot p_{13}^{*} \Delta_{X}^{-}= & p_{12}^{*} \Delta_{X}^{+} \cdot p_{23}^{*} \Delta_{X}^{-} \\
& p_{2}^{*} b \cdot p_{23}^{*} \Delta_{X}^{-}=0
\end{aligned}
$$

hence

$$
p_{12}^{*} \Delta_{X}^{-} \cdot p_{13}^{*} \Delta_{X}^{-}=p_{12}^{*}\left(\Delta_{X}^{+}-2 p_{2}^{*} b\right) \cdot p_{23}^{*} \Delta_{X}^{-} \text {. }
$$

Proof. We compute the left hand side of (17); we have:

$$
\begin{gathered}
p_{12}^{*} \Delta_{X}^{-} \cdot p_{13}^{*} \Delta_{X}^{-}=p_{12}^{*}\left(\Delta_{X}-\Gamma_{i}\right) \cdot p_{13}^{*}\left(\Delta_{X}-\Gamma_{i}\right) \\
=p_{12}^{*} \Delta_{X} \cdot p_{13}^{*} \Delta_{X}-p_{12}^{*} \Delta_{X} \cdot p_{13}^{*} \Gamma_{i}-p_{12}^{*} \Gamma_{i} \cdot p_{13}^{*} \Delta_{X}+p_{12}^{*} \Gamma_{i} \cdot p_{13}^{*} \Gamma_{i} .
\end{gathered}
$$

We observe now that

$$
\begin{gathered}
p_{12}^{*} \Delta_{X} \cdot p_{13}^{*} \Delta_{X}=p_{12}^{*} \Delta_{X} \cdot p_{23}^{*} \Delta_{X}, \quad p_{12}^{*} \Delta_{X} \cdot p_{13}^{*} \Gamma_{i}=p_{12}^{*} \Delta_{X} \cdot p_{23}^{*} \Gamma_{i}, \\
p_{12}^{*} \Gamma_{i} \cdot p_{13}^{*} \Delta_{X}=p_{12}^{*} \Gamma_{i} \cdot p_{23}^{*} \Gamma_{i}, \quad p_{12}^{*} \Gamma_{i} \cdot p_{13}^{*} \Gamma_{i}=p_{12}^{*} \Gamma_{i} \cdot p_{23}^{*} \Delta_{X} .
\end{gathered}
$$

It thus follows that

$$
p_{12}^{*} \Delta^{-} \cdot p_{13}^{*} \Delta^{-}=p_{12}^{*} \Delta_{X} \cdot p_{23}^{*} \Delta_{X}-p_{12}^{*} \Delta_{X} \cdot p_{23}^{*} \Gamma_{i}-p_{12}^{*} \Gamma_{i} \cdot p_{23}^{*} \Gamma_{i}+p_{12}^{*} \Gamma_{i} \cdot p_{23}^{*} \Delta_{X} .
$$

The right hand side of (20) is clearly equal to

$$
\left(p_{12}^{*} \Delta_{X}+p_{12}^{*} \Gamma_{i}\right) \cdot\left(p_{23}^{*} \Delta_{X}-p_{23}^{*} \Gamma_{i}\right),
$$

which is by definition $p_{12}^{*} \Delta_{X}^{+} \cdot p_{23}^{*} \Delta_{X}^{-}$, thus proving formula (17).

In order to prove formula (18), we use the fact that the 0 -cycle $b$ can be written as $\sum_{j} n_{j} x_{j}$, where the $x_{j}$ 's are $i$-invariant. By linearity, it thus suffices to prove (18) when $b$ is an $i$-invariant point of $X$. Now we have

$$
p_{2}^{*} b \cdot p_{23}^{*} \Delta^{-}=p_{23}^{*}((b, b)-(b, i b))=0 .
$$


Proof of Theorem 4.1. By (6), we have to prove that

$$
p_{1, \ldots, 2 m-1 *}\left(\Gamma^{1,2 m-1}(X, b)\right)=0 \text { in } \mathrm{CH}_{n}\left(X^{2 m-1}\right)_{\mathbb{Q}} .
$$

Now, by (5) and (16), using

$$
2 b=\pi^{*} a, \Delta_{X}^{+}=\pi_{2}^{*} \Delta_{Y}
$$

we get:

$$
2^{2 m-1} \Gamma^{1,2 m-1}(X, b)=p_{01}^{*}\left(\pi_{2}^{*} \Delta_{Y}^{a}+\Delta_{X}^{-}\right) \cdot \ldots \cdot p_{0,2 m-1}^{*}\left(\pi_{2}^{*} \Delta_{Y}^{a}+\Delta_{X}^{-}\right) .
$$

Here we use the notation

$$
\Delta_{Y}^{a}=\Delta_{Y}-p_{2}^{*} a \in \mathrm{CH}_{n}(Y \times Y)_{\mathbb{Q}},
$$

so that we have $\Delta_{X}^{+}-2 p_{2}^{*} b=\pi_{2}^{*} \Delta_{Y}^{a}$ and (19) can be written as

$$
p_{12}^{*} \Delta_{X}^{-} \cdot p_{13}^{*} \Delta_{X}^{-}=p_{12}^{*}\left(\pi_{2}^{*} \Delta_{Y}^{a}\right) \cdot p_{23}^{*} \Delta_{X}^{-} .
$$

our assumption $\Gamma^{m}(Y, a)=0$ on $Y$ can be written using Proposition 2.2 as

$$
q_{01}^{*} \Delta_{Y}^{a} \cdot q_{02}^{*} \Delta_{Y}^{a} \cdot \ldots \cdot q_{0 m}^{*} \Delta_{Y}^{a}=0 \text { in } \mathrm{CH}_{n}\left(Y^{m+1}\right)_{\mathbb{Q}},
$$

where the $q_{0 i}: Y^{m+1} \rightarrow Y \times Y$ are the projectors onto the product of the first and $i+1$-th factors.

Denote by $\pi_{r}: X^{r} \rightarrow Y^{r}$. We then clearly have for any $r$

$$
\pi_{r+1}^{*}\left(q_{01}^{*} \Delta_{Y}^{a} \cdot \ldots \cdot q_{0 r}^{*} \Delta_{Y}^{a}\right)=p_{01}^{*}\left(\pi_{2}^{*} \Delta_{Y}^{a}\right) \cdot \ldots \cdot p_{0 r}^{*}\left(\pi_{2}^{*} \Delta_{Y}^{a}\right) \text { in } \operatorname{CH}\left(X^{r}\right),
$$

and similarly for any choice of indices $i_{1}, \ldots, i_{r}$ in $\{1, \ldots, 2 m-1\}$. Developing now the product in (21), we get a sum of monomials which up to reordering the factors, take the form

$$
p_{01}^{*}\left(\pi_{2}^{*} \Delta_{Y}^{a}\right) \cdot \ldots \cdot p_{0 r}^{*}\left(\pi_{2}^{*} \Delta_{Y}^{a}\right) \cdot p_{0, r+1}^{*} \Delta_{X}^{-} \cdot \ldots \cdot p_{0,2 m-1}^{*} \Delta_{X}^{-}
$$

for some $r$. These terms vanish for $r \geq m$ by (24) and (23).

We now conclude the proof as follows: The terms $p_{0 i}^{*} \Delta_{X}^{-}$for $i \geq r+1$ can be grouped by pairs, and there are at least $\left\llcorner\frac{2 m-1-r}{2}\right\lrcorner$ such pairs. By (22), for each such pair, we have

$$
p_{0 i}^{*} \Delta_{X}^{-} \cdot p_{0, i+1}^{*} \Delta_{X}^{-}=p_{0 i}^{*}\left(\pi_{2}^{*} \Delta_{Y}^{a}\right) \cdot p_{i, i+1}^{*} \Delta^{-} .
$$

Hence each such pair produces a summand $p_{0 i}^{*}\left(\pi_{2}^{*} \Delta_{Y}^{a}\right)$. In total we get in (25) at least $r+\left\llcorner\frac{2 m-1-r}{2}\right\lrcorner$ factors of the form $p_{0 j}^{*}\left(\pi_{2}^{*} \Delta_{Y}^{a}\right)$. Now we have $r+\left\llcorner\frac{2 m-1-r}{2}\right\lrcorner \geq m$ unless $r=0$, and it follows that (25) vanishes for $r \geq 1$. Hence we proved that the only possibly nonzero monomial of the form (25) in the developed product (21) is $p_{01}^{*}\left(\Delta_{X}^{-}\right) \cdot \ldots \cdot p_{0,2 m-1}^{*} \Delta_{X}^{-}$. Thus we proved that

$$
2^{2 m-1} \Gamma^{1,2 m-1}(X, b)=p_{01}^{*}\left(\Delta_{X}^{-}\right) \cdot \ldots \cdot p_{0,2 m-1}^{*} \Delta_{X}^{-} \text {in } \mathrm{CH}\left(X^{2 m}\right) .
$$

Let $i^{\prime}$ be the involution $(i, I d, \ldots, I d)$ acting on $X^{2 m}$. Observe that each cycle $p_{0 j}^{*} \Delta_{X}^{-}$is skew-invariant under $i^{\prime *}$. It follows from (26) that $p_{01}^{*}\left(\Delta_{X}^{-}\right) \cdot \ldots \cdot p_{0,2 m-1}^{*} \Delta_{X}^{-}$is skew-invariant under $i^{\prime *}$, hence also under $i_{*}^{\prime}=i^{\prime *}$. But as we have $p_{1, \ldots, 2 m-1} \circ i^{\prime}=p_{1, \ldots, 2 m-1}$, we have

$$
\begin{aligned}
\Gamma^{2 m-1}(X, b) & =p_{1, \ldots, 2 m-1 *}\left(\Gamma^{1,2 m-1}(X, b)\right)=p_{1, \ldots, 2 m-1 *} \circ i_{*}^{\prime}\left(\Gamma^{1,2 m-1}(X, b)\right) \\
& =-p_{1, \ldots, 2 m-1 *}\left(\Gamma^{1,2 m-1}(X, b)\right)=-\Gamma^{2 m-1}(X, b),
\end{aligned}
$$

so that $\Gamma^{2 m-1}(X, b)=0$ in $\mathrm{CH}_{n}\left(X^{2 m-1}\right)$. 
We now turn to the proof of Theorem 1.3, (ii) and (iii) : in fact, the result will take the following more precise form:

Theorem 4.4. Let $\pi: X \rightarrow Y$ be a finite morphism of degree $d$. If $\Gamma^{m}(Y, a)=0$ in $\mathrm{CH}\left(Y^{m}\right) / R$ for some adequate equivalence relation $R$, and $b=\frac{1}{d} p^{*}$ a satisfies

$$
b * b=\Delta_{*}(b) \text { in } \mathrm{CH}_{0}(X \times X) / R,
$$

where $\Delta: X \rightarrow X \times X$ is the diagonal inclusion map, then $\Gamma^{d(m-1)+1}(X, b)=0$ in $\mathrm{CH}\left(X^{d m}\right) / R$.

Statement (ii) of Theorem 1.3 is the case where $R$ is rational equivalence (that is $R=0$ ) and $b$ is the class of a point of $X$, as all points satisfy (27) modulo rational equivalence. Statement (iii) of Theorem 1.3 is the case where $R$ is algebraic equivalence. Indeed, Theorem 4.4 applies since the equality $b * b=\Delta_{*}(b)$ in $\mathrm{CH}_{0}(X \times X)$ modulo algebraic equivalence is satisfied by 0 -cycles of degree 1 on a connected variety.

We first introduce some notation. Let as above $\Delta_{Y}^{a}:=\Delta_{Y}-p_{2}^{*} a \in \mathrm{CH}_{n}(Y \times Y)$ and similarly $\Delta_{X}^{b}:=\Delta_{X}-p_{2}^{*} b \in \mathrm{CH}_{n}(X \times X)$. In both expressions, $p_{2}$ is the projection from $Y \times Y$, resp. $X \times X$ onto its second factor. The proof of Theorem 4.4 will use the following result (which will replace formula (17) used previously when $d=2$ ):

Proposition 4.5. The morphism $\pi: X \rightarrow Y$ and the 0-cycle $b$ being as in Theorem 4.4. there exist cycles $\Gamma_{i} \in \mathrm{CH}^{(d-1) n}\left(X^{d+1}\right)$ such that

$$
\prod_{i=1}^{d} p_{0 i}^{*} \Delta_{X}^{b}=\sum_{i} p_{0 i}^{*}\left(\pi_{2}^{*} \Delta_{Y}^{a}\right) \cdot p_{0, D \backslash\{i\}}^{*} \Gamma_{i} \text { in } \mathrm{CH}^{n d}\left(X^{d+1}\right) / R,
$$

where $D$ is the set $\{1, \ldots, d\}$ and as usual $p_{0, D \backslash\{i\}}$ is the projection onto the product of the factors indexed by the set $\{0\} \cup D \backslash\{i\}$.

Before giving the proof, we will first prove a similar statement of independent interest for $\Delta_{X}$ and $\Delta_{Y}$, instead of $\Delta_{X}^{b}$ and $\Delta_{Y}^{a}$, as the proof is much simpler to write and we will use similar but slightly more involved arguments to prove Proposition 4.5. Namely we have the following result:

Proposition 4.6. Let $\pi: X \rightarrow Y$ be a finite morphism of degree $d$. There exist cycles $\Gamma_{i}^{\prime} \in \mathrm{CH}^{n(d-1)}\left(X^{d+1}\right)$ such that

$$
\prod_{i=1}^{d} p_{0 i}^{*} \Delta_{X}=\sum_{i} p_{0 i}^{*}\left(\pi_{2}^{*} \Delta_{Y}\right) \cdot p_{0, D \backslash\{i\}}^{*} \Gamma_{i}^{\prime} \text { in } \mathrm{CH}^{n d}\left(X^{d+1}\right) .
$$

Proof. Indeed, let us denote by $E_{k} \subset \mathrm{CH}\left(X^{k+1}\right)$ the ideal generated by the elements $p_{0 i}^{*}\left(\pi_{2}^{*} \Delta_{Y}\right), i=1, \ldots, k$. Next let

$$
\Sigma_{1}:=\pi_{2}^{-1}\left(\Delta_{Y}\right)-\Delta_{X} \in \mathrm{CH}(X \times X) .
$$

Note that, because $\pi$ is finite of degree $d, \Sigma_{1}$ is the class of the Zariski closure in $X \times X$ of the subvariety $\left\{\left(x, x_{1}\right) \in X^{0} \times X^{0}, \pi\left(x_{1}\right)=\pi(x), x_{1} \neq x\right\}$ where $X^{0}:=\pi^{-1}\left(Y^{0}\right)$ and $Y^{0}$ is the open set of $Y$ over which $\pi$ is étale of degree $d$. The first projection $p r_{1}: \Sigma_{1} \rightarrow X$ has degree $d-1$. Let us denote more generally by $\Sigma_{k} \subset X^{k+1}$ the Zariski closure in $X^{k+1}$ of the subvariety

$$
\left\{\left(x, x_{1}, \ldots, x_{k}\right) \in\left(X^{0}\right)^{k+1}, \pi\left(x_{i}\right)=\pi(x), x_{i} \neq x_{j} \text { for } i \neq j, x_{i} \neq x \text { for all } i\right\} .
$$

The contents of formula (29) is that $\prod_{i=1}^{d} p_{0 i}^{*} \Delta_{X}$ belongs to $E_{d}$. It is therefore a consequence of the following statement: 
Claim 4.7. For any integer $k \geq 1$, one has

$$
\alpha_{k} \prod_{i=1}^{k} p_{0 i}^{*} \Delta_{X}=\Sigma_{k} \text { in } \mathrm{CH}\left(X^{k+1}\right) / E_{k+1},
$$

with $\alpha_{k}=(-1)^{k} k$ !. In particular, $\prod_{i=1}^{d} p_{0 i}^{*} \Delta_{X}=0$ in $\mathrm{CH}\left(X^{d+1}\right) / E_{d}$.

The second statement follows from the first since $\Sigma_{d}$ is empty. The first statement is proved by induction on $k$. For $k=1$, the result is (30). The induction step is immediate: we have the following equalities in $\mathrm{CH}\left(X^{k+1}\right)$ :

$$
\begin{array}{r}
\prod_{i=1}^{k+1} p_{0 i}^{*} \Delta_{X}=p_{0, \ldots, k}^{*}\left(\prod_{i=1}^{k} p_{0 i}^{*} \Delta_{X}\right) \cdot p_{0, k+1}^{*} \Delta_{X} \\
=-\left(\prod_{i=1}^{k} p_{0 i}^{*} \Delta_{X}\right) \cdot p_{0, k+1}^{*} \Sigma_{1} \bmod E_{k+1} \\
=-\frac{1}{\alpha_{k}} p_{0, \ldots, k}^{*}\left(\Sigma_{k}\right) \cdot p_{0, k+1}^{*} \Sigma_{1} \bmod E_{k+1} .
\end{array}
$$

On the other hand, we observe that $\Sigma_{k+1}$ is obtained from $p_{0, \ldots, k}^{*}\left(\Sigma_{k}\right) \cdot p_{0, k+1}^{*} \Sigma_{1}$ by removing in the fibered product the components where $x_{k+1}$ equals one of the $x_{i}$ 's for $i=1, \ldots, k$. This gives rise to the following identity:

$$
p_{0, \ldots, k}^{*}\left(\Sigma_{k}\right) \cdot p_{0, k+1}^{*} \Sigma_{1}=\Sigma_{k+1}+\sum_{i=1}^{k} p_{0, \ldots, k}^{*}\left(\Sigma_{k}\right) \cdot p_{i, k+1}^{*} \Delta_{X} .
$$

In the right hand side of (34), we can replace (using again the induction hypothesis) $\Sigma_{k}$ by $\alpha_{k} \prod_{j=1}^{k} p_{0 j}^{*} \Delta_{X} \bmod E_{k}$ and we also observe that

$$
\prod_{j=1}^{k} p_{0 j}^{*} \Delta_{X} \cdot p_{i, k+1}^{*} \Delta_{X}=\prod_{i=1}^{k+1} p_{0 i}^{*} \Delta_{X}
$$

for any $i=1, \ldots, k$. Hence we get, using (33), (34) and (35),

$$
\prod_{i=1}^{k+1} p_{0 i}^{*} \Delta_{X}=-\frac{1}{\alpha_{k}} \Sigma_{k+1}-k \prod_{i=1}^{k+1} p_{0 i}^{*} \Delta_{X}
$$

This finally provides

$$
\alpha_{k+1} \prod_{i=1}^{k+1} p_{0 i}^{*} \Delta_{X}=\Sigma_{k+1}
$$

with $\alpha_{k+1}=-(k+1) \alpha_{k}$.

Proof of Proposition 4.5. We follow the above argument with $\Delta_{X}, \Delta_{Y}$ replaced by $\Delta_{X}^{b}$ and $\Delta_{Y}^{a}$, in order to prove Lemma 4.8 below. We use the following notation: we will work with the $n$-cycle $\Sigma_{k}^{b}$ of $X^{k+1}$ obtained by replacing formally in the definition (31) of $\Sigma_{k}$ each $x_{i}$ by $x_{i}-b$ and developing multilinearly. More rigorously, $\Sigma_{k}$ admits morphisms $p, p_{i}: \Sigma_{k} \rightarrow X$, obtained by restricting the projections $X^{k+1} \rightarrow X$ (where the factors are indexed by $\{0, \ldots, k\}$ and $\left.p=p_{0}\right)$. Let $\Gamma_{i} \subset \Sigma_{k} \times X$ be the graphs of these projections. Then we can obviously define $\Sigma_{k} \subset X^{k+1}$ as $\left(p, p r_{X^{k}}\right)_{*}\left(\prod_{i=1}^{k} p r_{\Sigma_{k}, i}^{*} \Gamma_{i}\right)$, where

- $p r_{X^{k}}: \Sigma_{k} \times X^{k} \rightarrow X^{k}$ is the second projection and $\left(p, p r_{X^{k}}\right): \Sigma_{k} \times X^{k} \rightarrow X^{k+1}$ is the obvious morphism. 
- $\operatorname{pr}_{\Sigma_{k}, i}: \Sigma_{k} \times X^{k} \rightarrow \Sigma_{k} \times X$ is the projection on the product of the first factor and the $i$-th factor of $X^{k}$.

On the other hand, we also have in $\Sigma_{k} \times X$ the graph $\Sigma_{k} \times\{b\}$ of the constant morphism mapping to $b$ if $b$ is a point, or more generally the $n$-cycle $p r_{X}^{*} b$ if $b$ is any 0 -cycle of degree 1. We then define analogously $\Sigma_{k}^{b}$ as follows:

$$
\Sigma_{k}^{b}=\left(p, p r_{X^{k}}\right)_{*}\left(\prod_{i=1}^{k} p r_{\Sigma_{k}, i}^{*}\left(\Gamma_{i}-p r_{X}^{*} b\right)\right) \text { in } \mathrm{CH}\left(X^{k+1}\right) .
$$

Developing the product above, we see that the formula for $\Sigma_{k}^{b}$ is of the form

$$
\Sigma_{k}^{b}=\sum_{I \subset\{1, \ldots, k\}}(-1)^{k-i} \lambda_{k, i, d} p_{0, I}^{*} \Sigma_{i} \cdot p_{J}^{*} b^{* j} \in \mathrm{CH}_{n}\left(X^{k+1}\right),
$$

where in the formula above, $I \sqcup J=\{1, \ldots, k\}, i=|I|$, and the $\lambda_{k, j, d}$ are combinatorial coefficients given by the formula

$$
\lambda_{k, i, d}=(d-i-1)(d-i-2) \ldots(d-k) .
$$

Indeed, the reason for (38) is the fact that the projection map

$$
p_{0, I}: \Sigma_{k} \rightarrow \Sigma_{i} \subset X^{i+1}
$$

has degree $(d-i-1)(d-i-2) \ldots(d-k)$. Note in particular, that $\Sigma_{k}^{b}=0$ for $k \geq d$. Next we define $E_{k, a, R} \subset \mathrm{CH}\left(X^{k+1}\right) / R$ as the ideal generated by the $p_{0, i}^{*} \Delta_{Y}^{a}$ for $i=1, \ldots, k$. Recall that $\Gamma^{1, k}(X, b)=\prod_{i=1}^{k} p_{0 i}^{*} \Delta_{X}^{b}$.

Lemma 4.8. The morphism $\pi: X \rightarrow Y$ and the 0 -cycle $b$ being as in Theorem 4.4, for any integer $k \geq 1$, one has

$$
\alpha_{k} \Gamma_{1, k}(X, b)=\Sigma_{k}^{b} \text { in } \mathrm{CH}\left(X^{k+1}\right) / E_{k, a, R},
$$

Proof. We have by (37), (38)

$$
\Delta_{X}^{b}=\pi_{2}^{*} \Delta_{Y}^{a}-\Sigma_{1}^{b},
$$

which can be written as $\Delta_{X}^{b}=-\Sigma_{1}^{b} \bmod E_{1, a, R}$, proving the case $k=1$. Assume the formula is proved for $k$. Then we have

$$
\begin{array}{r}
p_{0,1, \ldots, k}^{*} \Sigma_{k}^{b} \cdot p_{0, k+1}^{*} \Sigma_{1}^{b}=-\alpha_{k} p_{0,1, \ldots, k}^{*} \Gamma^{1, k}(X, b) \cdot p_{0, k+1}^{*} \Delta_{X}^{b} \\
=-\alpha_{k} \Gamma^{1, k+1}(X, b) \text { in } \mathrm{CH}\left(X^{k+1}\right) / E_{k, b, R} .
\end{array}
$$

Next we claim that we have the following relation in $\mathrm{CH}\left(X^{k+2}\right) / R$ :

$$
\begin{aligned}
p_{0,1, \ldots, k}^{*} \Sigma_{k}^{b} \cdot p_{0, k+1}^{*} \Sigma_{1}^{b}=\Sigma_{k+1}^{b}+ & \sum_{i=1}^{k} p_{0, \ldots, k}^{*} \Sigma_{k}^{b} \cdot p_{i, k+1}^{*} \Delta_{X}^{b} \\
& -\sum_{i=1}^{k} p_{0, \ldots, \hat{i}, k+1}^{*} \Sigma_{k}^{b} \cdot p_{i}^{*} b .
\end{aligned}
$$

This relation uses in a crucial way the identity

$$
\Delta_{*} b=p_{1}^{*} b \cdot p_{2}^{*} b \text { in } \mathrm{CH}_{0}(X \times X) / R .
$$

The beginning

$$
p_{0,1, \ldots, k}^{*} \Sigma_{k}^{b} \cdot p_{0, k+1}^{*} \Sigma_{1}^{b}=\Sigma_{k+1}^{b}+\sum_{i=1}^{k} p_{0, \ldots, k}^{*} \Sigma_{k}^{b} \cdot p_{i, k+1}^{*} \Delta_{X}^{b}+\ldots
$$


of the formula (41) is easily understood: it expresses the fact that in the left hand side, we include all possible $x_{k+1} \neq x$, while in $\Sigma_{k+1}^{b}$, we have to take into account the restriction $x_{k+1} \neq x_{i}$ for $i=1, \ldots, k$. The last term in (41) is explained as follows. The intersection with $p_{i, k+1}^{*} \Delta_{X}^{b}=p_{i, k+1}^{*} \Delta_{X}-p_{k+1}^{*} b$ produces a term

$$
\begin{gathered}
\Delta_{*}\left(x_{i}-b\right)-\left(x_{i}-b, b\right)=\left(x_{i}, x_{i}\right)-\Delta_{*} b-\left(x_{i}, b\right)+p_{i}^{*} b p_{k+1}^{*} b \\
=\left(x_{i}, x_{i}\right)-\left(x_{i}, b\right)
\end{gathered}
$$

on the product of the $i$ th and $k+1$ th factors. On the other hand, we had on the left in (41) the term

$$
\left(x_{i}-b, x_{i}-b\right)=\left(x_{i}, x_{i}\right)-\left(x_{i}, b\right)-\left(b, x_{i}\right)+(b, b)
$$

on the product of the $i$ th and $k+1$ th factors, which is unwanted in the development of $\Sigma_{k+1}^{b}$. Hence we also have to add on the right the extra term $-\left(b, x_{i}-b\right)$ on the product of the $i$ th and $k+1$ th factors, which is exactly the meaning of the term $-p_{0, \ldots, \hat{i}, k+1}^{*} \Sigma_{k}^{b} \cdot p_{i}^{*} b$. Thus the claim is proved.

Combined with (40) and the inductive assumption, (41) gives

$$
\begin{array}{r}
-\alpha_{k} \Gamma^{1, k+1}(X, b)=\Sigma_{k+1}^{b} \\
+\alpha_{k}\left(\sum_{i=1}^{k} p_{0, \ldots, k}^{*} \Gamma^{1, k}(X, b) \cdot p_{i, k+1}^{*} \Delta_{X}^{b}-\sum_{i=1}^{k} p_{0, \ldots, \hat{i}, k+1}^{*} \Gamma^{1, k}(X, b) \cdot p_{i}^{*} b\right) .
\end{array}
$$

The equality above holds in $\mathrm{CH}\left(X^{k+2}\right) / E_{k+1, a, R}$. Let us now prove that for any $i$,

$$
p_{0, \ldots, k}^{*} \Gamma^{1, k}(X, b) \cdot p_{i, k+1}^{*} \Delta_{X}^{b}-p_{0, \ldots, \hat{i}, k+1}^{*} \Gamma^{1, k}(X, b) \cdot p_{i}^{*} b=\Gamma^{1, k+1}(X, b)
$$

in $\mathrm{CH}\left(X^{k+2}\right) / R$. As

$$
\Gamma^{1, k}(X, b)=\prod_{i=1}^{k} p_{0 i}^{*} \Delta_{X}^{b}, \Gamma^{1, k+1}(X, b)=\prod_{i=1}^{k+1} p_{0 i}^{*} \Delta_{X}^{b},
$$

it clearly suffices to show that the cycles $p_{01}^{*} \Delta_{X}^{b} \cdot p_{12}^{*} \Delta_{X}^{b}-p_{1}^{*} b \cdot p_{02}^{*} \Delta_{X}^{b}$ and $p_{01}^{*} \Delta_{X}^{b} \cdot p_{02}^{*} \Delta_{X}^{b}$ of $X^{3}$ are equal in $\mathrm{CH}\left(X^{3}\right) / R$. We have

$$
\begin{gathered}
p_{01}^{*} \Delta_{X}^{b} \cdot p_{12}^{*} \Delta_{X}^{b}-p_{1}^{*} b \cdot p_{02}^{*} \Delta_{X}^{b}=\left(p_{01}^{*} \Delta_{X}-p_{1}^{*} b\right) \cdot\left(p_{12}^{*} \Delta_{X}-p_{2}^{*} b\right)-p_{1}^{*} b \cdot p_{02}^{*} \Delta_{X}+p_{1}^{*} b \cdot p_{2}^{*} b \\
=p_{01}^{*} \Delta_{X} \cdot p_{12}^{*} \Delta_{X}-p_{01}^{*} \Delta_{X} \cdot p_{2}^{*} b-p_{1}^{*} b \cdot p_{12}^{*} \Delta_{X}+p_{1}^{*} b \cdot p_{2}^{*} b-p_{1}^{*} b \cdot p_{02}^{*} \Delta_{X}+p_{1}^{*} b \cdot p_{2}^{*} b \\
=p_{01}^{*} \Delta_{X} \cdot p_{02}^{*} \Delta_{X}-p_{01}^{*} \Delta_{X} \cdot p_{2}^{*} b-p_{1}^{*} b \cdot p_{02}^{*} \Delta_{X}+p_{1}^{*} b \cdot p_{2}^{*} b
\end{gathered}
$$

in $\mathrm{CH}\left(X^{3}\right) / R$ because we assumed $p_{1}^{*} b \cdot p_{12}^{*} \Delta_{X}=p_{1}^{*} b \cdot p_{2}^{*} b$ in $\mathrm{CH}\left(X^{3}\right) / R$ (cf. (27)). On the other hand,

$$
\begin{gathered}
p_{01}^{*} \Delta_{X}^{b} \cdot p_{02}^{*} \Delta_{X}^{b}=\left(p_{01}^{*} \Delta_{X}-p_{1}^{*} b\right) \cdot\left(p_{02}^{*} \Delta_{X}-p_{2}^{*} b\right) \\
=p_{01}^{*} \Delta_{X} \cdot p_{02}^{*} \Delta_{X}-p_{01}^{*} \Delta_{X} \cdot p_{2}^{*} b-p_{1}^{*} b \cdot p_{02}^{*} \Delta_{X}+p_{1}^{*} b \cdot p_{2}^{*} b .
\end{gathered}
$$

Hence we proved that both terms in (44) are equal; using (43), we then get:

$$
-\alpha_{k} \Gamma^{1, k+1}(X, b)=\Sigma_{k+1, b}+\alpha_{k}\left(\sum_{i=1}^{k} \Gamma^{1, k+1}(X, b)\right),
$$

hence

$$
-(k+1) \alpha_{k} \Gamma^{1, k+1}(X, b)=\Sigma_{k+1, b} \text { in } \mathrm{CH}\left(X^{k+2}\right) / R
$$

and Lemma 4.8 is proved. 
Finally, Lemma 4.8 for $k=d$ implies Proposition 4.5 since $\Sigma_{d}^{b}=0$.

Proof of Theorem 4.4. By Lemma4.5 applied to each set of $d$ indices $\{1, \ldots, d\},\{d+1, \ldots, 2 d\}$, $\{(m-2) d+1, \ldots,(m-1) d\}$, we can write $\prod_{i=1}^{d(m-1)} p_{0 i}^{*} \Delta_{X}^{b}$ as a sum of products of $m-1$ cycles, each of them being of the form $p_{0 i}^{*}\left(\pi_{2}^{*} \Delta_{Y}^{a}\right) \cdot \Gamma^{\prime \prime}$ for an adequate index $i$ (one in each of the sets above). We now apply Proposition 2.5 to both $Y$ and $X$. Thus the assumption $\Gamma^{m}(Y, a)=0$ implies that for some cycle $\Gamma_{Y}$ on $Y^{m}$,

$$
\prod_{i=1}^{m-1} p_{0 i}^{*} \Delta_{Y}^{a}=p_{0}^{*} a \cdot \Gamma_{Y} \text { in } \mathrm{CH}\left(Y^{m}\right) / R
$$

Applying this relation to each product of $m-1$ factors $\prod_{k=1}^{m-1} p_{0 i_{k}}^{*}\left(\pi_{2}^{*} \Delta_{Y}^{a}\right)$ for adequate indices $i_{k}$ appearing above, we conclude that

$$
\Gamma^{1, d(m-1)}(X, b)=\prod_{i=1}^{d(m-1)} p_{0 i}^{*} \Delta_{X}^{b}=p_{0}^{*} b \cdot \Gamma_{X} \text { in } \mathrm{CH}\left(X^{d(m-1)+1}\right) / R
$$

for some cycle $\Gamma_{X}$ on $X^{d(m-1)+1}$. By Proposition 2.5 and using the fact that $b$ satisfies property (27), (that is, condition (10) in Proposition 2.5), we conclude that $\Gamma^{d(m-1)+1}(X, b)=0$ in $\mathrm{CH}\left(X^{d(m-1)+1}\right) / R$.

\subsection{Case of curves}

A special case of Theorem 1.3 (iii) is the case where $Y=\mathbb{P}^{1}$, so $X$ is a $d$-gonal curve. We then get the vanishing $\Gamma^{d+1}(X, b)=0$ in $\mathrm{CH}_{1}\left(X^{d+1}\right) / \mathrm{alg}$, where $b$ is any point of $X$. Recall now the Beauville decomposition of cycles on an abelian variety $A$ modulo rational or algebraic equivalence:

$$
\mathrm{CH}_{i}(A)=\oplus_{s} \mathrm{CH}_{i}(A)_{s},
$$

with

$$
\mathrm{CH}_{i}(A)_{s}:=\left\{z \in \mathrm{CH}_{i}(A), \mu_{k *} z=k^{2 i+s} z \text { for all } k \in \mathbb{Z}^{*}\right\}
$$

and similarly for Chow groups modulo algebraic equivalence. Here $\mu_{k}: A \rightarrow A$ is the morphism $a \mapsto k a$. Let now $X$ be a smooth genus $g$ projective curve and $A:=J(X) . X$ has an embedding in $J(X)$ which is canonical up to translation, hence determines a 1-cycle $Z$ in $J(X)$, well defined modulo algebraic equivalence. Thus we have a Beauville decomposition

$$
Z=\sum_{s} Z_{s} \text { in } \mathrm{CH}_{1}(A) / \text { alg. }
$$

For nonvanishing results concerning the cycles $Z_{s}$ (when $X$ is very general) and its decomposition, let us mention [7], [17] (in the later paper, it is proved that if $g \geq s^{2} / 2$, then $Z_{s} \neq 0$ modulo algebraic equivalence for a very general curve $X$ of genus $g$ ).

Let us show the following:

Proposition 4.9. The vanishing of $\Gamma^{d+1}(X, b)$ in $\mathrm{CH}_{1}\left(X^{d+1}\right) / \operatorname{alg}$ is equivalent to the vanishing of $Z_{s}, \forall s \geq d-1$, in $\mathrm{CH}_{1}(J(X)) /$ alg.

Proof. It suffices to prove the result for $d \leq g-1$, because we know by Theorem 1.3, (iii) (see Remark 1.5) that $\Gamma^{d+1}(X, b)=0$ in $\mathrm{CH}_{1}\left(X^{d+1}\right) /$ alg for some $d \leq g-1$. Assuming the proposition proved for $d \leq g-1$, this implies that $Z_{s}=0$ in $\mathrm{CH}_{1}(J(X)) /$ alg for all $s \geq g-1$, and thus for $d \geq g$, both vanishing statements are true.

We thus assume $d \leq g-1$; note that the cycle $\Gamma^{d+1}(X, b)$ is a 1-cycle of $X^{d+1}$ which is invariant under the action of the symmetric group $\mathfrak{S}_{d+1}$, so that its vanishing in $\mathrm{CH}_{1}\left(X^{d+1}\right) / \operatorname{alg}$ 
is equivalent to the vanishing of its image $\bar{\Gamma}^{d+1}(X, b)$ in $\mathrm{CH}_{1}\left(X^{(d+1)}\right) /$ alg. We now consider the inclusion

$$
\begin{gathered}
b_{g-d-1}: X^{(d+1)} \rightarrow X^{(g)} \\
z \mapsto z+(g-d-1) b
\end{gathered}
$$

and claim that $\bar{\Gamma}^{d+1}(X, b)=0$ in $\mathrm{CH}_{1}\left(X^{(d+1)}\right) /$ alg if and only if $b_{g-d-1 *}\left(\bar{\Gamma}^{d+1}(X, b)\right)=0$ in $\mathrm{CH}_{1}\left(X^{(g)}\right) / \mathrm{alg}$. Indeed, there is an incidence correspondence

$$
\Sigma \subset X^{(d+1)} \times X^{(g)}, \Sigma=\left\{\left(z, z^{\prime}\right), z^{\prime}=z+z^{\prime \prime} \text { for some } z^{\prime \prime} \in X^{(g-d-1)}\right\} .
$$

It is not hard to see that, due to its special form, the cycle $\bar{\Gamma}^{d+1}(X, b)$ satisfies

$$
\Sigma^{*}\left(b_{g-d-1 *}\left(\bar{\Gamma}^{d+1}(X, b)\right)\right)=\bar{\Gamma}^{d+1}(X, b),
$$

which proves the claim.

The next step is to observe that the Griffiths group of 1-cycles homologous to 0 modulo algebraic equivalence is a birational invariant. This is elementary to show using resolution of indeterminacies of birational maps, as it is invariant under blow-up and is functorial under pushforward and pullbacks under generically finite morphisms. As $X^{(g)}$ is birational to $J(X)$ via the Abel map, we conclude that $\bar{\Gamma}^{d+1}(X, b)=0$ in $\mathrm{CH}_{1}\left(X^{(d+1)}\right) /$ alg if and only if its image $W$ in $J(X)$ under the Abel map vanishes in $\mathrm{CH}_{1}(J(X)) /$ alg.

Finally, we observe that a cycle appearing in the formula (11) for $\Gamma^{d+1}(X, b)$, which is up to permutation of the form

$$
\{(x, \ldots, x, b, \ldots, b), x \in X\},
$$

where $x$ appears $k$ times and $b$ appears $d+1-k$ times, maps under the Abel map to a 1-cycle of $J(X)$ algebraically equivalent to $\mu_{k *}(Z)$. The vanishing of $W$ in $\mathrm{CH}_{1}(J(X)) /$ alg thus gives

$$
\sum_{k=1}^{d+1}(-1)^{d+1-k}\left(\begin{array}{c}
d+1 \\
k
\end{array}\right) \mu_{k *} Z=0 \text { in } \mathrm{CH}_{1}(J(X)) / \text { alg. }
$$

Writing the Beauville decomposition

$$
Z=\sum_{s} Z_{s}
$$

the vanishing of $W$ in $\mathrm{CH}_{1}(J(X)) /$ alg is equivalent to

$$
\sum_{k=1}^{d+1}(-1)^{d+1-k}\left(\begin{array}{c}
d+1 \\
k
\end{array}\right) k^{2+s} Z_{s}=0, \text { in } \mathrm{CH}_{1}(J(X)) / \operatorname{alg}
$$

for any $s$.

We now have the following easy lemma:

Lemma 4.10. We have $\sum_{k=1}^{d+1}(-1)^{d+1-k}\left(\begin{array}{c}d+1 \\ k\end{array}\right) k^{2+s}=0$ for $s \leq d-2$, and

$$
\sum_{k=1}^{d+1}(-1)^{d+1-k}\left(\begin{array}{c}
d+1 \\
k
\end{array}\right) k^{2+s} \neq 0
$$

for $s \geq d-1$.

This shows that the vanishing (46) is equivalent to the vanishing of $Z_{s}$ for $s \geq d-1$.

Remark 4.11. Proposition 4.9 is also proved in 11, where it is used to deduce the vanishing $\Gamma^{g+2}(X, a)=0$ of Remark 1.4 for any point $a \in X$, from the main result of Colombo and van Geemen [5]. 


\section{$5 \quad$ Hyper-Kähler manifolds}

\subsection{Proof of Theorem 1.9}

We prove in this section the following theorem (cf. Theorem 1.9 of the introduction):

Theorem 5.1. Let $S$ be a $K 3$ surface, and let $X=S^{[n]}$. Then

$$
\Gamma^{2 n+1}\left(X, o_{X}\right)=0 \text { in } \mathrm{CH}_{2 n}\left(X^{2 n+1}\right),
$$

where $o_{X}$ is the canonical 0 -cycle on $X$ constructed from the canonical 0-cycle of $S$.

Here the cycle $o_{S}$ appears in the following theorem from [2] providing a list of relations which hold in the Chow ring of a self-product of a $K 3$ surface.

Theorem 5.2. Let $S$ be a smooth projective $K 3$ surface. Then there is a degree 1 zerocycle $o_{S} \in \mathrm{CH}_{0}(S)$ satisfying the following equalities (which are all polynomial relations in $\mathrm{CH}\left(S^{k}\right)$ for adequate $k$, between the cycles $\left.p_{i}^{*} o_{S}, p_{j}^{*} L, p_{s t}^{*} \Delta_{S}\right)$ :

1. $L^{2}-\operatorname{deg}\left(L^{2}\right) o_{S}=0$ in $\mathrm{CH}_{0}(S)$, for any $L \in P i c S$.

2. $\Delta_{S} \cdot p_{1}^{*} L-L \times o_{S}-o_{S} \times L=0$ in $\mathrm{CH}_{1}(S \times S)$ for any $L \in \operatorname{Pic} S$, where $p_{1}$ is the first projection from $S \times S$ to $S$, and $L \times o_{S}=p_{1}^{*} L \cdot p_{2}^{*} o_{S}$.

3. $\Gamma^{3}\left(S, o_{S}\right)=0$ in $\mathrm{CH}_{2}(S \times S \times S)$. (Using formula (1) and the identity $\Delta_{3}=p_{12}^{*} \Delta_{S}$. $p_{13}^{*} \Delta_{S}$, we can also see Property 3 as a polynomial relation in $\mathrm{CH}\left(S^{3}\right)$ involving the classes $p_{i j}^{*} \Delta_{S}$ and $p_{k}^{*}\left(o_{S}\right)$.)

4. $\Delta_{S}^{2}=24 p_{1}^{*} o_{S} \cdot p_{2}^{*} o_{S}$ in $\mathrm{CH}_{0}(S \times S)$.

5. $\Delta_{S} \cdot p_{1}^{*} o_{S}-p_{1}^{*} o_{S} \cdot p_{2}^{*} o_{S}=0$ in $\mathrm{CH}_{0}(S \times S)$.

Note that property $\left[\right.$ is (27) and is easily satisfied because $o_{S}$ is the class of a point in $S$. Property 4 is a consequence of Property 3 which implies $c_{2}(S)=24 o_{S}$ in $\mathrm{CH}_{0}(S)$, and Property 5 .

Remark 5.3. The above relations are the nontrivial relations involving $p_{i}^{*}\left(o_{S}\right), p_{j}^{*} L, L \in$ $\mathrm{CH}^{1}(S)$ and the $p_{k l}^{*} \Delta_{S}$ and with the property that in at least one monomial, an index is repeated. To make a complete list of such relations, one should add the "trivial relations", which hold on any surface, namely

1. $o_{X} \cdot L=0$ in $\mathrm{CH}(S), L \in \mathrm{CH}^{1}(S)$,

2. $o_{X} \cdot o_{X}=0$ in $\mathrm{CH}(S)$,

3. $p_{12}^{*} \Delta_{S} \cdot p_{23}^{*} \Delta_{S}=p_{13}^{*} \Delta_{S} \cdot p_{23}^{*} \Delta_{S}$ in $\mathrm{CH}(S \times S \times S)$.

As in [16, the ingredients of the proof of Theorem 5.1] are 1) the results of de CataldoMigliorini [4, which will allow, thanks to Proposition [5.6, to translate the problem into computations in ordinary self-products $S^{N}, N \leq(2 n+1) n$, of a $K 3$ surface; 2$)$ the relations listed in Theorem 5.2, 3) the recent result of Yin [20. The latter says basically that for a regular surface $S$, the cohomological polynomial relations on $S^{N}$ between the diagonal classes and the pull-back under the various projections of the class of a point are generated by the relations listed above (or rather, their cohomological counterpart) and the Kimura relation (cf. [10, [18, 3.2.3]) which holds when the motive of $S$ is finite dimensional. A key point of the proof will be thus the fact that the Kimura relation is not needed to express the pull-back to $S^{N}$ of the vanishing relation $\left[\Gamma^{2 n+1}\left(X, o_{X}\right)\right]=0$.

We first recall some notation related to $S^{n}$ and $S^{[n]}$, for any smooth surface $S$. Let $\mu=\left\{A_{1}, \ldots, A_{l}\right\}, l=: l(\mu)$ be a partition of $\{1, \ldots, n\}$, where all the $A_{i}$ 's are nonempty. Let $S^{\mu} \cong S^{l(\mu)} \subset S^{n}$ be the set

$$
\left\{\left(s_{1}, \ldots, s_{n}\right), s_{i}=s_{j} \text { if } i, j \in A_{k} \text { for some } k\right\} .
$$


The image $\overline{S^{(\mu)}}$ of $S^{\mu}$ in $S^{(n)}$ is a stratum of $S^{(n)}$. It is not normal in general, but its normalization $S^{(\mu)}$ is the quotient of $S^{\mu}$ by the subgroup $\mathfrak{S}_{\mu}$ of $\mathfrak{S}_{n}$ preserving $S^{\mu}$, that is acting on $\{1, \ldots, n\}$ by permuting the $A_{i}$ 's with the same cardinality. Let $c: S^{[n]} \rightarrow S^{(n)}$ be the Hilbert-Chow morphism and let $E_{\mu}:=S^{\mu} \times_{S^{(n)}} S^{[n]} \subset S^{\mu} \times S^{[n]}$. It is known that $E_{\mu}$ is irreducible of dimension $n+l(\mu)$. We see $E_{\mu}$ as a correspondence between $S^{\mu}$ and $S^{[n]}$.

Theorem 5.4. (de Cataldo-Migliorini [4]) The collection $\left(E_{\mu}\right)_{\mu}$ of correspondences identifies the motive of $S^{[n]}$ to a submotive of the disjoint union $\sqcup_{\mu} S^{\mu}$. More precisely, for some combinatorial coefficients $\lambda_{\mu}$,

$$
\Delta_{X}=\sum_{\mu} \lambda_{\mu}\left(E_{\mu}, E_{\mu}\right)_{*}\left(\Delta_{S^{\mu}}\right) \text { in } \mathrm{CH}_{2 n}(X \times X) .
$$

The result above implies in particular:

Corollary 5.5. Let $X:=S^{[n]}$. For any integer $k$, the map

$$
\oplus_{\left(\mu_{1}, \ldots, \mu_{k}\right)}\left(E_{\mu_{1}}, \ldots, E_{\mu_{k}}\right)^{*}: \mathrm{CH}^{*}\left(X^{k}\right) \rightarrow \oplus_{\left(\mu_{1}, \ldots, \mu_{k}\right)} \mathrm{CH}^{*}\left(S^{\mu_{1}} \times \ldots \times S^{\mu_{k}}\right)
$$

is injective.

We now have the following result: Let $n$ and $k$ be fixed. Let us denote by $\Delta_{k} \subset X^{k}$ the small diagonal of $X^{k}$, where $X:=S^{[n]}$, for a smooth projective surface $S$.

Proposition 5.6. For any $k$-uple $\left(\mu_{1}, \ldots, \mu_{k}\right)$ of partitions of $\{1, \ldots, n\}$, there exists a universal (i.e. independent of $S$ ) polynomial $P_{\mu}$. (in many variables) with the following property: For any smooth quasi-projective surface $S$,

$$
\left(E_{\mu_{1}}, \ldots, E_{\mu_{k}}\right)^{*}\left(\Delta_{k}\right)=P_{\mu}\left(p r_{i}^{*} c_{2}(S), p r_{j}^{*}\left(K_{S}\right), p r_{s t}^{*}\left(\Delta_{S}\right)\right) \text { in } \mathrm{CH}\left(S^{\mu_{1}} \times \ldots \times S^{\mu_{k}}\right),
$$

where the $\mathrm{pr}_{i}$ 's are the projections from $\prod_{i} S^{\mu_{i}} \cong S^{N}$ to its factors (isomorphic to $S$ ), and the $p r_{s t}$ 's are the projections from $\prod_{i} S^{\mu_{i}}$ to the products of two of its factors (isomorphic to $S \times S)$.

Proof. Proposition 5.6 is a particular case of Theorem 5.12 whose proof will be sketched in Subsection 5.2 and will be completed in [19, because the cycles $\left(E_{\mu_{1}}, \ldots, E_{\mu_{k}}\right)^{*}\left(\Delta_{k}\right) \in$ $\mathrm{CH}\left(S^{N}\right)$ are clearly universally defined cycles in the sense of Definition 5.11. Indeed, for any family $\mathcal{S} \rightarrow B$ of smooth quasi-projective surfaces, we can construct the smooth family of relative Hilbert schemes $\mathcal{X}:=\mathcal{S}^{[n / B]}$ and its relative small diagonals

$$
\Delta_{k / B}(\mathcal{X}) \subset \mathcal{X}^{k / B} .
$$

Then we have the relative correspondences $E_{\mu_{i}} \subset \mathcal{S}^{\mu / B} \times_{B} \mathcal{X}$, which are proper over the first summand, and we have thus the relative cycle

$$
E_{\mu .}^{*}\left(\Delta_{k / B}(\mathcal{X})\right) \in \mathrm{CH}\left(\mathcal{S}^{[N / B]}\right), N=l\left(\mu_{1}\right)+\ldots+l\left(\mu_{k}\right),
$$

satisfying the functoriality properties stated in Definition 5.11 because the morphisms $E_{\mu_{i}} \rightarrow B$ are flat.

Remark 5.7. One may have the feeling that the canonical class is not necessary in Proposition [5.6] as set theoretically one wants the set of $\left(s_{1}, \ldots, s_{k}\right) \in S^{\mu_{1}} \times \ldots \times S^{\mu_{k}}$ such that there is a subscheme $x \in S^{[n]}$ whose associated cycle is $s_{i}$ (or rather its image in $S^{(n)}$ ) and this does not seem to involve the intrinsic geometry of $S$, except for the self-intersection of the diagonal, thus only $c_{2}$. In fact, due to excess formulas, the canonical class actually appears, as the simplest example shows: Let $X$ be $S^{[2]}, k=3$, and $\mu_{1}=\mu_{2}=\mu_{3}$ be the partition of $\{1,2\}$ consisting of a single set with 2 elements. Then $E_{\mu_{1}}=E_{\mu_{2}}=E_{\mu_{3}}=E$ is the exceptional divisor of $S^{[2]}$ and we have

$$
\left(E_{\mu_{1}}, E_{\mu_{2}}, E_{\mu_{3}}\right)^{*}\left(\Delta_{3}\right)=\Delta_{*}\left(p_{*}\left(E_{\mid E}^{2}\right)\right),
$$

where $\Delta: S \rightarrow S^{3}$ is the diagonal inclusion, and $p: E \rightarrow S$ is the natural map. But $p_{*}\left(E_{\mid E}^{2}\right) \in \mathrm{CH}^{1}(S)$ is a nonzero multiple of the canonical class of $S$. 
Remark 5.8. We proved in [16] similar statement where instead of the small diagonal, arbitrary polynomials in the Chern classes of the tautological sheaf on $X^{[n]}$ and the Chern classes of the ideal sheaf of the incidence correspondence in $S^{[n-1]} \times S$ ) are considered; the same kind of arguments used in loc. cit., which are in fact borrowed from [6], can be applied to prove Proposition 5.6. but the proofs are very intricated and lengthy and in fact all these results can also be obtained as Proposition 5.6. as a consequence of Theorem 5.12.

We now show how Theorem 5.1 follows from Proposition 5.6

Proof of Theorem [5.1. We have to prove the vanishing of $\Gamma^{2 n+1}\left(X, o_{X}\right)$, where $S$ is a smooth projective $K 3$ surface and $X=S^{[n]}$. By Corollary 5.5 it suffices to show that for any $2 n+1$ uple $\left(\mu_{1}, \ldots, \mu_{2 n+1}\right)$ of partitions of $\{1, \ldots, n\}$, we have

$$
\left(E_{\mu_{1}}, \ldots, E_{\mu_{2 n+1}}\right)^{*}\left(\Gamma^{2 n+1}\left(X, o_{X}\right)\right)=0
$$

in $\mathrm{CH}\left(S^{\mu_{1}} \times \ldots \times S^{\mu_{2 n+1}}\right)$. As $\Gamma^{2 n+1}\left(X, o_{X}\right)$ is a combination of cycles which up to permutation of factors are of the form $\Delta_{k} \times o_{X}^{2 n+1-k}$ and $E_{\mu}^{*} o_{X}=0$ if $\mu \neq\{\{1\}, \ldots,\{n\}\}$, and is equal to $n !\left(o_{S}, \ldots, o_{S}\right)$ if $\mu=\{\{1\}, \ldots,\{n\}\}$, it follows from Proposition 5.6 that there exists a polynomial $Q_{\mu}$. (in many variables) with the following property: For any smooth projective surface $S$, and any point $o_{S} \in S$,

$$
\left(E_{\mu_{1}}, \ldots, E_{\mu_{2 n+1}}\right)^{*}\left(\Gamma^{2 n+1}\left(X, o_{X}\right)\right)=Q_{\mu}\left(p r_{i}^{*} c_{2}(S), p r_{j}^{*}\left(K_{S}\right), p r_{l}^{*} o_{S}, p r_{s t}^{*}\left(\Delta_{S}\right)\right)
$$

in $\mathrm{CH}\left(S^{\mu_{1}} \times \ldots \times S^{\mu_{2 n+1}}\right)$. We know by [12, Proposition 1.3] (see also Theorem 1.2] (i)) that for any regular surface $S$, and any point $o_{S} \in S, \Gamma^{2 n+1}\left(X, o_{X}\right)$ is cohomologous to 0 , where $o_{X}$ is any point of $X=S^{[n]}$ over $n o_{S} \in S^{(n)}$. It follows that for each $2 n+1$-uple $\left(\mu_{1}, \ldots, \mu_{2 n+1}\right)$ as above, the cycle

$$
\left(E_{\mu_{1}}, \ldots, E_{\mu_{2 n+1}}\right)^{*}\left(\Gamma^{2 n+1}\left(X, o_{X}\right)\right)
$$

is cohomologous to 0 in $S^{\mu_{1}} \times \ldots \times S^{\mu_{2 n+1}}$. Hence the polynomial $Q_{\mu}$. has the property that for a regular surface $S$,

$$
Q_{\mu .}\left(\operatorname{pr}_{i}^{*}\left[c_{2}(S)\right], \operatorname{pr}_{j}^{*}\left(\left[K_{S}\right]\right), \operatorname{pr}_{l}^{*}\left[o_{S}\right], \operatorname{pr}_{s t}^{*}\left(\left[\Delta_{S}\right]\right)\right)=0 \text { in } H^{*}\left(S^{\mu_{1}} \times \ldots \times S^{\mu_{2 n+1}}, \mathbb{Q}\right) .
$$

Here the brackets denote the cohomology class of the corresponding cycles. In this equation, we can of course replace $\left[c_{2}(S)\right]$ by $\chi_{t o p}(S)\left[o_{S}\right]$, with $\chi_{t o p}(S)$ determined by the polynomial relation (this is relation 4 in Theorem [5.2) $\left[\Delta_{S}\right]^{2}=\chi_{t o p}(S) p r_{1}^{*}\left[o_{S}\right] \cup p r_{2}^{*}\left[o_{S}\right]$ in $H^{4}(S \times S, \mathbb{Q})$. We now follow [16] (see also [20]): The cohomological version of the equations given in Theorem 5.2 with $L=K_{S}$ holds on any smooth projective surface with $b_{1}=0$, and if the canonical class satisfies $\left[K_{S}\right]=0$ or $\left[K_{S}\right]^{2} \neq 0$, one can reduce modulo these relations any polynomial expression in the variables

$$
\operatorname{pr}_{i}^{*}[p t], \operatorname{pr}_{j}^{*}\left[K_{S}\right], p r_{s t}^{*}\left[\Delta_{S}\right]
$$

to a linear combination of monomials in the variables $p r_{i}^{*}[p t], p r_{j}^{*}\left[K_{S}\right], p r_{s t}^{*}\left[\Delta_{S}\right]^{0}$, with the property that no index appears twice in the monomial. Here the class $\left[\Delta_{S}\right]^{0}$ is the class

$$
\left[\Delta_{S}\right]-p r_{1}^{*}[p t]-p r_{2}^{*}[p t]-\lambda p r_{1}^{*}\left[K_{S}\right] \cup p r_{2}^{*}\left[K_{S}\right],
$$

where the coefficient $\lambda$, when $K_{S} \neq 0$, is determined by the relation $\lambda\left[K_{S}\right]^{2}=1$ (the class $\left[\Delta_{S}\right]^{0} \in H^{4}(S \times S, \mathbb{Q})$ ) is the projector onto $\left.H^{2}(S, \mathbb{Q})^{\perp\left[K_{S}\right]}\right)$. Now, it is clear by Künneth decomposition that if a linear combination of such monomials vanishes in $H^{*}\left(S^{N}, \mathbb{Q}\right)=$ $H^{*}(S, \mathbb{Q})^{\otimes N}$, then for fixed distinct indices $i_{1}, \ldots, i_{m}, j_{1}, \ldots, j_{p}, k_{1}, \ldots, k_{q}$, the sum of such monomials of the form

$$
p r_{i_{1}}^{*}[p t] \cdot \ldots \cdot p r_{i_{m}}^{*}[p t] \cdot p r_{j_{1}}^{*}\left[K_{S}\right] \cdot \ldots \cdot p r_{j_{p}}^{*}\left[K_{S}\right] \cdot p r_{k_{1}}^{*} 1_{S} \cdot \ldots \cdot p r_{k_{q}}^{*} 1_{S} \cdot \prod_{s_{1}, t_{1}, \ldots, s_{l}, t_{l}} p r_{s_{i}, t_{i}}^{*}\left[\Delta_{S}\right]^{0}
$$


where the indices $s_{i}, t_{j}$ exhaust the remaining indices, are all distinct and are different from the $i_{s}, j_{s}, k_{s}$, has to be 0 . This way, we reduced the problem to linear combinations of monomials of the form

$$
p r_{s_{1} t_{1}}^{*}\left[\Delta_{S}\right]^{0} \cdot \ldots \cdot p r_{s_{l} t_{l}}^{*}\left[\Delta_{S}\right]^{0}
$$

on $S^{2 l}$, where no index is repeated. We now have the following result due to Yin [20]: The "Kimura relation" is a relation between monomials of the above type. It says that, for $M=\operatorname{dim} H^{2}(S, \mathbb{Q})^{\perp\left[K_{S}\right]}$, the cohomology class of the projector onto $\bigwedge^{M+1} H^{2}(S, \mathbb{Q})^{\perp\left[K_{S}\right]} \subset$ $H^{2 M+2}\left(S^{M+1}, \mathbb{Q}\right)$ is 0 , which is obvious since $\bigwedge^{M+1} H^{2}(S, \mathbb{Q})^{\perp\left[K_{S}\right]}=0$. The class of this projector is the class

$$
\sum_{\sigma \in \mathfrak{S}_{M+1}} \epsilon(\sigma) \prod_{i=1}^{M+1} p r_{i, M+1+\sigma(i)}^{*}\left[\Delta_{S}\right]^{0} \in H^{4 M+4}\left(S^{2 M+2}, \mathbb{Q}\right) .
$$

and the Kimura relation is thus the vanishing of (52).

Theorem 5.9. (Yin, [20]) For any integer $m$, the relations in $H^{*}\left(S^{m}, \mathbb{Q}\right)$ between the monomials (51) with no repeated indices are generated by the pull-back to $S^{m}$ of the Kimura relation via a projection (and a permutation) $S^{m} \rightarrow S^{2 N+2}$.

We deduce the following

Corollary 5.10. The polynomial $Q_{\mu}$. belongs to the ideal generated by the trivial relations (see Remark 5.3), the relation $c_{2}(S)=\chi_{\text {top }}(S) o_{S}$ (where we recover $\chi_{\text {top }}(S)$ as the selfintersection of $\Delta_{S}$ ) and the relations listed in Theorem 5.2 with $L=K_{S}$.

Proof. Indeed, choose for $S$ a smooth projective surface with $b_{1}(S)=0$ and $b_{2}(S)>\frac{n(2 n+1)}{2}$. Then by Theorem [5.9] there are no linear relations between the monomials (51) with no repeated index if $s \leq(2 n+1) n$. On the other hand, we have the vanishing of the cohomology class

$$
Q_{\mu .}\left(p r_{i}^{*}\left[c_{2}(S)\right], p r_{j}^{*}\left[K_{S}\right], \operatorname{pr}_{l}^{*}\left[o_{S}\right], p r_{s t}^{*}\left[\Delta_{S}\right]\right) \in H^{*}\left(S^{N}, \mathbb{Q}\right),
$$

where $N=\sum_{i} l\left(\mu_{i}\right) \leq(2 n+1) n$. It then follows from the above reduction that the polynomial $Q_{\mu}$., where one substitutes $\chi_{t o p}(S)\left[o_{S}\right]$ to $\left[c_{2}(S)\right]$, belongs to the ideal generated by the cohomological version of the relations given in Theorem 5.2 , with $L=K_{S}$.

The proof of Theorem 5.1 is now finished. Indeed, $S$ being now a $K 3$ surface, we know by Theorem 5.2 that the relation $\chi_{t o p}(S) o_{S}=c_{2}(S)$ holds in $C H_{0}(S)$ and that the relations listed in Theorem 5.2 hold in $\mathrm{CH}\left(S^{k}\right)$ for adequate $k$. As the polynomial $Q_{\mu}$., where one substitutes $\chi_{t o p}(S) o_{S}$ to $c_{2}(S)$, belongs to the ideal generated by the relations given in Theorem 5.2 and the trivial relations, we conclude that $Q_{\mu} .=0$ in $\mathrm{CH}\left(S^{N}\right)$. By (49), we proved the vanishing (48)

$$
\left(E_{\mu_{1}}, \ldots, E_{\mu_{2 n+1}}\right)^{*}\left(\Gamma^{2 n+1}\left(X, o_{X}\right)\right)=0 \text { in } \mathrm{CH}\left(S^{N}\right),
$$

which concludes the proof.

\subsection{Universally defined cycles}

This subsection is devoted to introducing the notion of "universally defined cycles" and to sketching the proof of a quite general statement which will be fully proved in [19]. It concerns "universally defined" cycles on self-products of surfaces. We first explain the meaning of this expression. In the following, we work with Chow groups with integral coefficients, and we will write $\mathrm{CH}(X)_{\mathbb{Q}}$ for cycles with $\mathbb{Q}$-coefficients. 
Definition 5.11. Let $n, N$ be integers. A universally defined cycle on the $N$-th power of smooth complex algebraic varieties $X$ of a given dimension $n$ consists in the following data: for each smooth family of $n$-dimensional algebraic varieties $\mathcal{X} \rightarrow B$, where $B$ is smooth quasiprojective, a cycle $z_{\mathcal{X}} \in \mathrm{CH}\left(\mathcal{X}^{N / B}\right)$ is given, satisfying the following conditions:

(i) If $r: B^{\prime} \rightarrow B$ is a morphism, with induced morphism

$$
R_{N}:\left(\mathcal{X}^{\prime}\right)^{N / B^{\prime}} \rightarrow \mathcal{X}^{N / B}, \mathcal{X}^{\prime}:=\mathcal{X} \times{ }_{B} B^{\prime},
$$

then

$$
z_{\mathcal{X}^{\prime}}=R_{N}^{*} z_{\mathcal{X}} \text { in } \mathrm{CH}\left(\left(\mathcal{X}^{\prime}\right)^{N / B^{\prime}}\right) .
$$

(ii) If $\mathcal{X} \rightarrow B$ is a family as above and $\mathcal{Y} \subset \mathcal{X}$ is a Zariski open set, then

$$
z_{\mathcal{Y}}=z_{\mathcal{X} \mid \mathcal{Y}^{N / B}} \text { in } \mathrm{CH}\left(\mathcal{Y}^{N / B}\right) \text {. }
$$

Theorem 5.12. For any universally defined cycle $z$ on $N$-th powers of surfaces, there exists a polynomial $P$ with rational coefficients, depending only on $z$, such that for any smooth algebraic surface $S$ defined over $\mathbb{C}$,

$$
z_{S}=P\left(p r_{i}^{*} c_{1}(S), p r_{j}^{*} c_{2}(S), p r_{r s}^{*} \Delta_{S}\right) \text { in } \mathrm{CH}\left(S^{N}\right)_{\mathbb{Q}} .
$$

Remark 5.13. One could introduce as well universally defined cycles with $\mathbb{Q}$-coefficients, by replacing everywhere in the definition above $\mathrm{CH}$ by $\mathrm{CH}_{\mathbb{Q}}$. It is possible that the conclusion holds as well for universally defined cycles with $\mathbb{Q}$-coefficients, but our present proof uses the integral structure.

We will give some hints on the proof, with a complete proof only in the case $N=1$ (Proposition 5.18) and the construction of the desired polynomials (Corollary 5.15] and Proposition 5.17). We refer to 19 for a full treatment. Let us first show how to produce such polynomials. Let $G:=G(2,5)$ be the Grassmannian of 2-dimensional vector subspaces in $\mathbb{C}^{5}$. Any smooth complex projective surface can be embedded in $G$, for example by choosing 5 general sections of a very ample vector bundle $E$ on $S$. Let $\mathcal{O}_{G}(1)$ be the Plücker line bundle on $G$, and let $c \in \mathrm{CH}^{2}(G)$ be the second Chern class of the tautological rank 2 vector bundle on $G$. We choose an integer $d$, and consider the universal family $\mathcal{S}_{d} \rightarrow B$ of smooth surfaces in $G$ which are complete intersections of 4 members of $\left|\mathcal{O}_{G}(d)\right|$. The smooth variety $B$ is thus the vector space $H^{0}\left(G, \mathcal{O}_{G}(d)\right)^{4}$ and

$$
\mathcal{S}_{d} \subset \mathcal{S}_{d, \text { univ }}
$$

is the Zariski open set consisting of points where $\mathcal{S}_{\text {d,univ }} \rightarrow B$ is smooth. Here

$$
\mathcal{S}_{d, \text { univ }}:=\left\{(b, x) \in B \times G, b=\left(f_{1, b}, \ldots, f_{4, b}\right), f_{i, b}(x)=0 \forall i\right\} .
$$

There is an obvious morphism

$$
f: \mathcal{S}_{d} \rightarrow G
$$

given by the restriction to $\mathcal{S}_{d}$ of the second projection $\mathcal{S}_{d, \text { univ }} \rightarrow G$, which induces for any $N \geq 1$ the morphism

$$
f_{N}: \mathcal{S}_{d}^{N / U} \rightarrow G^{N}
$$

with induced pull-back morphism $f_{N}^{*}: \mathrm{CH}\left(G^{N}\right) \rightarrow \mathrm{CH}\left(\mathcal{S}_{d}^{N / U}\right)$. We now use the following result, which is one of the main ingredients in the proof of Theorem 5.12 .

Proposition 5.14. For any integer $N>0$ and sufficiently large $d, \mathrm{CH}\left(\mathcal{S}_{d}^{N / U}\right)$ is generated as a $\mathrm{CH}\left(G^{N}\right)$-module by the relative partial diagonals $\Delta_{I / U}\left(\mathcal{S}_{d}\right)$.

Here $I$ denotes as usual a partition of $\{1, \ldots, N\}$, determining a partial diagonal. 
Proof of Proposition 5.14 By the localization exact sequence, it suffices to prove the result with $\mathcal{S}_{d}$ replaced by $\mathcal{S}_{d, \text { univ }}$. Next consider the natural morphism

$$
f_{N}: \mathcal{S}_{d, \text { univ }}^{N / B} \rightarrow G^{d}
$$

The fiber of $f_{N}$ over a $N$-uple $\left(x_{1}, \ldots x_{N}\right)$ consists of the set of 4 -uples $\left(\sigma_{1}, \ldots, \sigma_{4}\right) \in$ $H^{0}\left(G, \mathcal{O}_{G}(d)\right)^{4}$ having the property that the $\sigma_{i}$ 's vanish at all points $x_{i}$. As $d$ is large compared to $N$, any $k$ distinct points of $G$ with $k \leq N$ impose independent conditions to $H^{0}\left(G, \mathcal{O}_{G}(d)\right)$, and thus, denoting by $G_{k}^{N}$ the locally closed subvariety of $G^{N}$ consisting of $N$-uples with exactly $k$-distinct points, which is the disjoint union of the diagonals $\Delta_{I}(G)$ with $l(I)=k$ (or rather of the $\Delta_{I}^{0}(G):=\Delta_{I}(G) \backslash \cup_{J, l(J)<k} \Delta_{J}(G)$ ), we find that $f_{N}^{-1}\left(G_{k}^{N}\right)$ is a Zariski open set in a vector bundle over $G_{k}^{N}$. It follows from the localization exact sequence and $A^{1}$-invariance that $\mathrm{CH}\left(G_{k}^{N}\right) \stackrel{f_{N}^{*}}{\rightarrow} \mathrm{CH}\left(f_{N}^{-1}\left(G_{k}^{N}\right)\right)$ is surjective. Writing $G^{N}$ as the disjoint union of the $\Delta_{I}^{0}(G)$, we conclude from the above and the localization exact sequence that

$$
\oplus_{I} \mathrm{CH}\left(\Delta_{I}(G)\right) \stackrel{\left(j_{I *} \circ f_{I}^{*}\right)}{\rightarrow} \mathrm{CH}\left(\mathcal{S}_{d, \text { univ }}^{N / B}\right)
$$

is surjective, where $f_{I}$ is the restriction of $f_{N}$ to $f_{N}^{-1}\left(\Delta_{I}(G)\right) \subset G^{N}$ and $j_{I}$ is the inclusion of $f_{N}^{-1}\left(\Delta_{I}(G)\right)$ in $\mathcal{S}_{d}^{N / U}$. Note that $f_{I}^{-1}\left(\Delta_{I}(G)\right)=\Delta_{I / B}\left(\mathcal{S}_{d, \text { univ }}\right)$. Finally, we observe that the restriction map

$$
\mathrm{CH}\left(G^{N}\right) \rightarrow \mathrm{CH}\left(\Delta_{I}(G)\right)
$$

is surjective, and that for any $\alpha \in \mathrm{CH}\left(G^{N}\right)$,

$$
j_{I *} \circ f_{I}^{*}\left(\alpha_{\mid \Delta_{I}(G)}\right)=f_{N}^{*} \alpha \cdot\left(j_{I *} \circ f_{I}^{*}\right)(1)=f_{N}^{*} \alpha \cdot \Delta_{I / B}\left(\mathcal{S}_{d, \text { univ }}\right),
$$

and this finishes the proof.

Corollary 5.15. For any universally defined cycle $z$ on $N$-th powers of surfaces and for sufficiently large $d$, there exists a polynomial $P_{d}$ with rational coefficients, depending only on $z$ and $d$ such that for any smooth complete intersection surface $S_{d} \subset G$ as above,

$$
z_{S_{d}}=P_{d}\left(p r_{i}^{*} c_{1}\left(S_{d}\right), p r_{j}^{*} c_{2}\left(S_{d}\right), p r_{r s}^{*} \Delta_{S_{d}}\right) \text { in } \mathrm{CH}\left(S_{d}^{N}\right)_{\mathbb{Q}} .
$$

Furthermore, $(4 d-5)^{2 N} P_{d}$ has integral coefficients.

Proof. As $z$ is universal, there exists a cycle $z_{\mathcal{S}_{d}} \in \mathrm{CH}\left(\mathcal{S}_{d}^{N / B}\right)$ such that for any surface $S_{d}$ as above,

$$
z_{S_{d}}=\left(z_{\mathcal{S}_{d}}\right)_{\mid S_{d}^{N}}
$$

where we see $S_{d}$ as a fiber of the universal family $\mathcal{S}_{d} \rightarrow B$. We next use Proposition 5.14 to write, for $d>>0, \mathcal{Z}_{d}$ as a combination $\sum_{I} f_{N}^{*} \alpha_{I} \cdot \Delta_{I / B}\left(\mathcal{S}_{d}\right)$, where $\alpha_{I} \in \mathrm{CH}(G(2,5))$. Furthermore, is it immediate to prove that $\mathrm{CH}\left(G^{N}\right)=\mathrm{CH}(G)^{\otimes N}$, so that we can write each $\alpha_{I}$ as a polynomial with integral coefficients in $p r_{i}^{*} m, m:=c_{1}\left(\mathcal{O}_{G}(1)\right)=c_{1}(E)$ and $p r_{j}^{*} c, c:=c_{2}(E)$ where $E$ is the dual of the tautological subbundle on $G$. Of course, under restriction to $S_{d}^{N}$, only polynomials of weighted degree $\leq 2$ in each set of variables $p r_{i}^{*} m, p r_{i}^{*} c$ will survive. We now observe that the restriction of $m$ to $S_{d}$ is a rational multiple of $c_{1}(S)$ (more precisely, $K_{S_{d}}=\mathcal{O}_{G}(4 d-5)_{\mid S}$ by the adjunction formula), and the restriction of $c$ to $S_{d}$ is an adequate linear combination of $\frac{1}{(4 d-5)^{2}} c_{1}\left(S_{d}\right)^{2}, c_{2}\left(S_{d}\right)$. Putting everything together and using the fact that the relative diagonals $\Delta_{I / B}\left(\mathcal{S}_{d}\right)$ restrict to $\Delta_{I}\left(S_{d}\right)$, we get the result.

Remark 5.16. Note that Corollary 5.15 is true more generally for the regular and complete intersection locus $S_{\text {reg }}$ of any set of 4 degree $d$ equations on $G$. The proof uses Proposition 5.14 (which works for the family $\mathcal{S}_{d} \rightarrow B$ of smooth complete intersection quasi-projective surfaces), and both conditions (i) and (ii) in Definition 5.11 . 
The corollary above proves Theorem 5.12 for smooth complete intersection surfaces of degree $d$, and more generally for the regular and complete intersection locus of any set of 4 degree $d$ equations on $G$. What remains to be done is to prove that the polynomial above works for all surfaces. Note that the polynomial $P_{d}$ is in fact not uniquely defined as only its value on the set of variables $p r_{i}^{*} c_{1}\left(S_{d}\right), p r_{j}^{*} c_{2}\left(S_{d}\right), p r_{s t}^{*} \Delta_{S_{d}}$ is well defined in $\mathrm{CH}\left(S_{d}^{N}\right)_{\mathbb{Q}}$. Hence a priori $P_{d}$ is only defined modulo the relations in $\mathrm{CH}\left(S_{d}^{N}\right)_{\mathbb{Q}}$ between these variables. However, the following result shows that a part of $P_{d}$ is in fact independent of $d$ for large $d$.

Proposition 5.17. For any universally defined cycle $z$ on $N$-th powers of surfaces, there exists a polynomial $Q$ in the variables $p_{s t}^{*} \Delta_{S}$, depending only on $z$, with the following property: For any smooth surface $S$, there is a Zariski dense open set $V \subset S$ such that $z_{V}=Q\left(p r_{s t}^{*} \Delta_{S}\right)$ in $\mathrm{CH}\left(V^{N}\right)_{\mathbb{Q}}$.

Proof. Let $Q_{d}$ be the part of the polynomial $P_{d}$ which involves only the diagonals. Then let $U_{d} \subset S_{d}$ be the complement of a hyperplane section defined by the choice of a codimension 2 vector subspace $W \subset \mathbb{C}^{5}$ in general position. As $c_{1}\left(\mathcal{O}_{S_{d}}(1)\right)$ and $c_{2}(E)$ vanish in $\mathrm{CH}\left(U_{d}\right)$, we deduce from (53) that

$$
z_{U_{d}}=Q_{d}\left(p r_{s t}^{*} \Delta_{U_{d}}\right) \text { in } \mathrm{CH}\left(U_{d}^{N}\right) .
$$

We observe now that for $d^{\prime} \leq d$, a surface $S_{d^{\prime}}$ which is the complete intersection in $G$ of hypersurfaces of degree $d^{\prime}$ is an irreducible component of a (singular) surface $\Sigma_{d}=S_{d^{\prime}} \cup T$ defined as the complete intersection in $G$ of four degree $d$ hypersurfaces containing $S_{d^{\prime}}$ and that, denoting $C:=S_{d^{\prime}} \cap T$, the open set $U_{d^{\prime}}^{\prime}:=S_{d^{\prime}} \backslash C$ is contained in the smooth locus of $\Sigma_{d}$. From Remark 5.16, we thus get that

$$
z_{U_{d^{\prime}}^{\prime}}=P_{d}\left(p r_{i}^{*} c_{1}\left(U_{d^{\prime}}^{\prime}\right), p r_{j}^{*} c_{2}\left(U_{d^{\prime}}^{\prime}\right), p r_{s t}^{*} \Delta_{U_{d^{\prime}}^{\prime}}\right) \text { in } \mathrm{CH}\left(U_{d^{\prime}}^{\prime}\right)_{\mathbb{Q}},
$$

and after restriction to $V_{d^{\prime}}:=U_{d^{\prime}} \cap U_{d^{\prime}}^{\prime}$, this becomes

$$
z_{V_{d^{\prime}}}=Q_{d}\left(p r_{s t}^{*} \Delta_{V_{d^{\prime}}}\right) \text { in } \mathrm{CH}\left(V_{d^{\prime}}^{N}\right)_{\mathbb{Q}}
$$

On the other hand, we also have (54) for $d^{\prime}$, which provides after restriction to $V_{d^{\prime}}$

$$
z_{V_{d^{\prime}}}=Q_{d^{\prime}}\left(p r_{s t}^{*} \Delta_{V_{d^{\prime}}}\right) \text { in } \mathrm{CH}\left(V_{d^{\prime}}^{N}\right)_{\mathbb{Q}} .
$$

Hence $Q_{d}-Q_{d^{\prime}}$ belongs to the kernel of the map

$$
\begin{gathered}
\mathrm{ev}_{d^{\prime}}: \mathbb{Q}\left[X_{r s}\right]_{1 \leq r \neq s \leq N} \rightarrow \mathrm{CH}\left(V^{N}\right), \\
f \mapsto f\left(p_{r s}^{*} \Delta_{V}\right),
\end{gathered}
$$

where $V$ is a sufficiently small Zariski open set of a general complete intersection of four hypersurfaces of degree $d^{\prime}$ in $G$. On the other hand, it follows from the above construction that $\operatorname{Kerev}_{d} \subset \operatorname{Kerev}_{d^{\prime}}$ for $d^{\prime} \leq d$. As the polynomials we consider are homogeneous of given degree (equal to half the codimension of $z$ ), they live in a finite dimensional vector space and we conclude that these kernels are in fact stationary, equal to $K$ for $d \geq d_{0}$. So we finally conclude that there exists a $d_{0}$ such that for $d \geq d^{\prime} \geq d_{0}, Q_{d}-Q_{d^{\prime}}$ belongs to $K$. It follows that for any $d$, for any reduced complete intersection of four degree $d$ hypersurfaces in $G$, and for a dense Zariski open set $V \subset S$

$$
z_{V}=Q_{d_{0}}\left(p r_{s t}^{*} \Delta_{V}\right) \text { in } \mathrm{CH}\left(V^{N}\right)_{\mathbb{Q}} .
$$

As any smooth quasi-projective surface has a dense Zariski open set which is contained in the smooth locus of such a complete intersection for $d$ large enough, the proposition is proved, with $Q=Q_{d_{0}}$. 
We finish this section with the proof of Theorem 5.12 in the case $N=1$.

Proposition 5.18. Let $z$ be a universally defined cycle on surfaces. Then there is a polynomial $P$ independent of $S$ and with integral coefficients, such that for any smooth quasiprojective surface $S$,

$$
z_{S}=P\left(c_{2}(S), c_{1}(S)\right) \in \mathrm{CH}(S)_{\mathbb{Q}} .
$$

Proof. Let us first treat the case of $z \in \mathrm{CH}^{1}(S)$ universally defined. For complete intersections $S_{d}$ of four hypersurfaces of degree $d$ in $G$, we must have by Corollary 5.15

$$
z=\alpha_{d} K_{S_{d}}
$$

for some rational number $\alpha_{d}$ such that $(4 d-5) \alpha_{d} \in \mathbb{Z}$, and for any surface $S$, choosing a very ample vector bundle $E$ of rank 2 on $S$ to embed $S$ in $G$, and choosing $d$ large enough, we get

$$
z_{S \mid U}=\alpha_{d} K_{U} \text { in } \mathrm{CH}^{1}(U)_{\mathbb{Q}},
$$

where $U=S \backslash C$, the surface $S \cup_{C} T=\Sigma_{d}$ being the complete intersection of four degree $d$ hypersurfaces containing $S$ in $G$. The curve $C$ belongs to the linear system $\left|(4 d-5) L-K_{S}\right|$, where $L=\operatorname{det} E=\mathcal{O}_{G}(1)_{\mid S}$. For a general choice of equations and $d$ large enough, the curve $C$ will be irreducible, so by the localization exact sequence, (59) rewrites as

$$
z_{S}=\alpha_{d} K_{S}+\beta_{d} C \text { in } \mathrm{CH}^{1}(S)_{\mathbb{Q}}
$$

If $K_{S}$ and $L$ are linearly independent in $\mathrm{CH}^{1}(S)_{\mathbb{Q}}$, this implies, because the left hand side is independent of $L$, that $\beta_{d}=0$ and thus $z_{S}=\alpha_{d} K_{S}$, with $\alpha_{d}=: \alpha$ necessarily independent of $d$. If not, we simply blow up $S$ at one point and choose $L$ on $\widetilde{S}$ linearly independent of $K_{\widetilde{S}}$ in $\mathrm{CH}^{1}(\widetilde{S})_{\mathbb{Q}}$. Then the above conclusion applies to $\widetilde{S}$, hence we get

$$
z_{\widetilde{S}}=\alpha K_{\widetilde{S}} \text { in } \mathrm{CH}^{1}(\widetilde{S}) .
$$

As

$$
S \backslash\{p\} \cong \widetilde{S} \backslash E_{p}, \mathrm{CH}^{1}(S)_{\mathbb{Q}} \cong \mathrm{CH}^{1}(S \backslash\{p\})_{\mathbb{Q}},
$$

(60) is also true for $S$ by condition (ii) in Definition [5.11. Finally $\alpha$ has to be an integer since $(4 d-5) \alpha \in \mathbb{Z}$ for any $d$. This proves the result for $z_{S} \in \mathrm{CH}^{1}(S)$ universally defined.

Let us now prove the result for a universally defined cycle $z_{S} \in \mathrm{CH}^{2}(S)$. We start the proof exactly as before, and we have by Corollary 5.15 and Remark 5.16 that for $S$ the regular locus of a complete intersection of four hypersurfaces of degree $d$ in $G, z_{S}=$ $\mu_{d} c_{2}(S)+\nu_{d} c_{1}(S)^{2}$ in $\mathrm{CH}_{0}(S)_{\mathbb{Q}}$. With the notation above, we conclude as before that for any pair $(S, E)$ consisting of a smooth surface $S$ and a very ample rank 2 vector bundle in a bounded family (depending on $d$ ), we have

$$
z_{S \mid U}=\mu_{d} c_{2}(S)_{\mid U}+\nu_{d} c_{1}(S)_{\mid U}^{2} \text { in } \mathrm{CH}_{0}(U)_{\mathbb{Q}}
$$

where $U=S \backslash C$ and the curve $C$ is defined as above. By the localization exact sequence, this is equivalent to

$$
z_{S}=\mu_{d} c_{2}(S)+\nu_{d} c_{1}(S)^{2}+z_{S}^{\prime} \text { in } \mathrm{CH}_{0}(S)_{\mathbb{Q}}
$$

where $z_{S}^{\prime}$ is a 0 -cycle supported on $C$. If $d$ is large enough (with respect to the given bounded family), the surface $S$ is schematically cut out by hypersurfaces of degree $d$ in $G$ and this implies that the morphism of sheaves

$$
I_{S}(d) \otimes \mathcal{O}_{S} \rightarrow N_{S / G}^{*}(d), N_{S / G}^{*}=\mathcal{I}_{S} / \mathcal{I}_{S}^{2},
$$

is surjective. The curve $C$ is the curve obtained as the degeneracy locus of the morphism

$$
f: \mathcal{O}_{S}^{4} \rightarrow N_{S / G}^{*}(d)
$$


deduced from the choice of 4 degree $d$ equations defining $S$ in $G$. It is in fact better to see such a curve as embedded in $\mathbb{P}\left(N_{S / G}(-d)\right)$, as the complete intersection of 4 hypersurfaces in the linear system $\left|\mathcal{O}_{\mathbb{P}\left(N_{S / G}(-d)\right)}(1)\right|$. Let $B_{S}$ be the Zariski open set of $I_{S}(d)^{4}$ consisting of the $\left(f_{1}, \ldots, f_{4}\right) \in I_{S}(d)^{4}$ such that the $f_{i}$ 's form a regular sequence cutting $S$ at its generic point. For each element $b \in B_{S}$, let $C_{b} \subset \mathbb{P}\left(N_{S / G}(-d)\right)$ be the corresponding curve. We have a family of complete intersection surfaces

$$
\mathcal{S}^{\prime} \rightarrow B_{S}
$$

which is a subfamily of the family $\mathcal{S}_{d}^{\prime} \rightarrow B$ of all complete intersection surfaces of four degree $d$ hypersurfaces in $G$. The family $\mathcal{S}^{\prime} \rightarrow B_{S}$ contains $B_{S} \times S$, which itself contains the family of curves $\mathcal{C} \rightarrow B_{S}$ with fiber $C_{b}$ over $b \in B_{S}$. The surfaces $b \times\left(S \backslash C_{b}\right)$ are contained the smooth locus of the surfaces $S_{b}^{\prime}$. In conclusion, the inclusion

$$
\mathcal{S}^{\prime} \subset \mathcal{S}_{d}^{\prime}
$$

of families of complete intersection surfaces restricts, by considering the Zariski open sets where the surfaces are smooth, to an open inclusion of smooth families of surfaces

$$
\left(B_{S} \times S\right) \backslash \mathcal{C} \hookrightarrow \mathcal{S}_{d} \times{ }_{B} B_{S}
$$

Applying the conditions (i) and (ii) in Definition 5.11 and Proposition 5.14, we get that

$$
\left(p r_{S}^{*}\left(\mu_{d} c_{2}(S)+\nu_{d} c_{1}(S)^{2}\right)\right)_{\mid\left(B_{S} \times S\right) \backslash \mathcal{C}}=\left(z_{B_{S} \times S}\right)_{\mid\left(B_{S} \times S\right) \backslash \mathcal{C}} \text { in } \mathrm{CH}\left(\left(B_{S} \times S\right) \backslash \mathcal{C}\right)_{\mathbb{Q}},
$$

which rewrites equivalently, by the localization exact sequence, as

$$
\left(p r_{S}^{*}\left(\mu_{d} c_{2}(S)+\nu_{d} c_{1}(S)^{2}\right)\right)-z_{B_{S} \times S}=i_{*}(w) \text { in } \mathrm{CH}\left(B_{S} \times S\right)_{\mathbb{Q}},
$$

where $w \in \mathrm{CH}^{1}(\mathcal{C})_{\mathbb{Q}}$ and $i: \mathcal{C} \rightarrow B_{S} \times S$ is the natural morphism. Here, as already mentioned, it is much better to consider the variant of $\mathcal{C}$ which is contained in $B_{S} \times \mathbb{P}\left(N_{S / G}(-d)\right)$, (and the morphism $i$ is only at the general point of $B_{S}$ an embedding) because it is then the universal complete intersection of four hypersurfaces and this allows to conclude with exactly the same proof as in Proposition 5.14.

Lemma 5.19. The restriction map $\mathrm{CH}^{1}\left(\mathbb{P}\left(N_{S / G}(-d)\right)\right) \rightarrow \mathrm{CH}^{1}(\mathcal{C})$ is surjective.

Note that

$$
\mathrm{CH}^{1}\left(\mathbb{P}\left(N_{S / G}(-d)\right)\right)=\mathrm{CH}^{1}(S) \oplus \mathbb{Z} c_{1}\left(\mathcal{O}_{\mathbb{P}\left(N_{S / G}(-d)\right)}(1)\right) .
$$

The cycle $w$ of (63) thus decomposes as $w=\left(w_{S}\right)_{\mid C}+\left(w_{P}\right)_{\mid C}$, with

$$
w_{P}=\alpha c_{1}\left(\mathcal{O}_{\mathbb{P}\left(N_{S / G}(-d)\right)}(1)\right) \in \mathrm{CH}^{1}\left(\mathbb{P}\left(N_{S / G}(-d)\right)\right)_{\mathbb{Q}} .
$$

As $C_{b} \subset \mathbb{P}\left(N_{S / G}(-d)\right)$ is the intersection of four members of the linear system $\left.\mid \mathcal{O}_{\mathbb{P}\left(N_{S / G}(-d)\right)}(1)\right) \mid$ for any given $b \in B_{S}$, we get

$$
i_{*}\left(\left(w_{P}\right)_{\mid C_{b}}\right)=\alpha s_{2}\left(N_{S / G}(-d)\right),
$$

where $s_{2}$ is the second Segre class. Note that by (171), $s_{2}\left(N_{S / G}(-d)\right)$ can be explicitly computed as a linear combination with integral coefficients of $L^{2}, c_{2}(E), c_{2}(S), c_{1}(S)^{2}$, involving non trivially $c_{2}(E)$. Thus we get from (63) and (64), by restricting to any point $b \in B_{S}$

$$
\left.z_{S}=\mu_{d} c_{2}(S)+\nu_{d} c_{1}(S)^{2}\right)-\alpha s_{2}\left(N_{S / G}(-d)\right)-\left((4 d-5) L-K_{S}\right) \cdot w_{S} \text { in } \mathrm{CH}_{0}(S)_{\mathbb{Q}} .
$$

Let us analyze in general the cycle $w_{S} \in \mathrm{CH}^{1}(S)$. We observe first that this cycle is universally defined for triples $(S, E, d)$, where $d$ has to be large and we conclude as in the previous proof that there are rational numbers $\gamma, \delta$ such that

$$
w_{S}=\gamma c_{1}(S)+\delta L
$$


where $L=c_{1}(E)$. Combining this with (65), we get, using the fact that the curve $i\left(C_{b}\right) \subset S$ belongs to the linear system $\left|(4 d-5) L-K_{S}\right|$ :

$$
\begin{array}{r}
z_{S}=\mu_{d} c_{2}(S)+\nu_{d} c_{1}(S)^{2}-\alpha s_{2}\left(N_{S / G}(-d)\right) \\
-\left((4 d-5) L-K_{S}\right) \cdot\left(\gamma K_{S}+\delta L\right) \text { in } \mathrm{CH}_{0}(S)_{\mathbb{Q}},
\end{array}
$$

where the coefficients $\alpha, \beta, \gamma$ a priori depend on $S, E$ and $d$. Finally we observe that the left hand side depends only on $S$, while in the right hand side, for fixed large $d$, we can freely change $c_{2}(E), L=c_{1}(E)$ (staying in a bounded family). Assume first that no nonzero linear combination of the cycles $s_{2}\left(N_{S / G}(-d)\right), L^{2}, K_{S} \cdot L$ belongs to the linear span of the cycles $z_{S}, c_{2}(S), c_{1}(S)^{2}$. Then (66) easily implies that $\alpha=\delta=\gamma=0$, so that we get in this case

$$
z_{S}=\mu_{d} c_{2}(S)+\nu_{d} c_{1}(S)^{2} \text { in } \mathrm{CH}_{0}(S)_{\mathbb{Q}} .
$$

To treat the general case, we will first prove the result assuming $\mathrm{CH}_{0}(S)$ is nontrivial, that is, if $S$ is projective and connected, $\mathrm{CH}_{0}(S) \neq \mathbb{Z}$. It then easily follows that $\mathrm{CH}_{0}(S)$ is uncountable, and one deduces immediately that for the blow-up $S^{\prime}$ of $S$ at 6 general points, and for adequate choice of $E^{\prime}$, (and thus $L^{\prime}=\operatorname{det} E^{\prime}$ ) no combination of the cycles $s_{2}\left(N_{S^{\prime} / G}(-d)\right), L^{\prime 2}, K_{S^{\prime}} \cdot L^{\prime}$ belongs to the linear span of the cycles $z_{S^{\prime}}, c_{2}\left(S^{\prime}\right), c_{1}\left(S^{\prime}\right)^{2}$. Thus we conclude from (67) that for a general blow-up $S^{\prime}$ of $S$ along 6 points,

$$
z_{S^{\prime}}=\mu_{d} c_{2}\left(S^{\prime}\right)+\nu_{d} c_{1}\left(S^{\prime}\right)^{2} \text { in } \mathrm{CH}_{0}\left(S^{\prime}\right)_{\mathbb{Q}}
$$

But this immediately implies that for the original surface $S$, (67) also holds, because we can restrict (68) to the Zariski open set $S^{\prime} \backslash \cup_{i=1}^{6} E_{i}=S \backslash\left\{p_{1}, \ldots, p_{6}\right\}$, where the $p_{i}$ 's are the points blown-up in $S$ and the $E_{i}$ 's are the exceptional curves over them. Using property (ii) in Definition [5.11] we find that (68) provides:

$$
\left(z_{S}\right)_{\mid S \backslash\left\{p_{1}, \ldots, p_{6}\right\}}=\mu_{d} c_{2}(S)+\nu_{d} c_{1}(S)^{2} \text { in } \mathrm{CH}_{0}\left(S \backslash\left\{p_{1}, \ldots, p_{6}\right\}\right)_{\mathbb{Q}} .
$$

As the $p_{i}$ 's are general points, this immediately implies that

$$
z_{S}=\mu_{d} c_{2}(S)+\nu_{d} c_{1}(S)^{2} \text { in } \mathrm{CH}_{0}(S)_{\mathbb{Q}} .
$$

Finally, the above discussion was concerning polarized surfaces in a bounded family (depending on $d$ ). Taking $d$ larger, we conclude that the coefficients $\mu_{d}$ and $\nu_{d}$ do not depend on $d$, which concludes the proof, under the assumption made on $\mathrm{CH}_{0}(S)$.

It now only remains to prove the result for surfaces with $\mathrm{CH}_{0}(S)=\mathbb{Z}$. In this case, we can use the following trick, using the fact that we already know that

$$
\mu_{d}=\mu, \nu_{d}=\nu
$$

are independent of $d$ (supposed to be large), so that

$$
z_{S}=\mu c_{2}(S)+\nu c_{1}(S)^{2}
$$

when $S$ is the regular locus of the complete intersection of four hypersurfaces of degree $d$ in $G$. Here $\mu, \nu$ are a priori rational numbers but in fact, they are integers because $(4 d-5)^{5} \mu_{d},(4 d-5)^{2} \nu_{d}$ are integers by Corollary 5.15. We observe now that with the notation introduced for Proposition 5.14 the equality

$$
z_{\mathcal{S}_{d}}=\mu c_{2}\left(K_{\mathcal{S}_{d} / B}\right)+\nu c_{1}\left(K_{\mathcal{S}_{d} / B}\right)^{2}
$$

must hold in $\mathrm{CH}^{2}\left(\mathcal{S}_{d}\right)$, because both sides are integral cycles, the equality is satisfied in $\mathrm{CH}^{2}\left(\mathcal{S}_{d}\right)_{\mathbb{Q}}$, and $\mathrm{CH}^{2}\left(\mathcal{S}_{d}\right)$ has no torsion. We choose as before a very ample rank 2 vector bundle $E$ on $S$ with $c_{2}(E)=c \in \mathrm{CH}_{0}(S)=\mathbb{Z}$, and embed $S$ in $G$ using 5 general sections of 
$E$. For large $d$, we then have as before the family $\left(B_{S} \times S\right) \backslash \mathcal{C} \subset \mathcal{S}_{d}$, where $B_{S}$ is a Zariski open set of $I_{S}(d)^{4}$, and we conclude by restriction, using (i) and (ii) of Definition 5.11 that

$$
\left(p r_{S}^{*} z_{S}\right)_{\mid\left(B_{S} \times S\right) \backslash \mathcal{C}}=\left(\mu p r_{S}^{*} c_{2}\left(K_{S}\right)+\nu p r_{S}^{*} c_{1}\left(K_{S}\right)^{2}\right)_{\mid\left(B_{S} \times S\right) \backslash \mathcal{C}}
$$

holds in $\mathrm{CH}^{2}\left(\left(B_{S} \times S\right) \backslash \mathcal{C}\right)$. In other words, we proved the same equalities as before, but in $\mathrm{CH}$ instead of $\mathrm{CH}_{\mathbb{Q}}$. We now apply the localization exact sequence and Lemma 5.19 to conclude that (65) holds in fact with integral cycles, namely

$$
z_{S}=\mu c_{2}(S)+\nu c_{1}(S)^{2}-\alpha s_{2}\left(N_{S / G}(-d)\right)-\left((4 d-5) L-K_{S}\right) \cdot w_{S} \text { in } \mathrm{CH}_{0}(S),
$$

where $\alpha$ is an integer and $w_{S} \in \mathrm{CH}^{1}(S)$.

We now choose $d, c$ and $L=\operatorname{det} E$ in such a way that an arbitrarily large given integer $M$ divides both $s_{2}\left(N_{S / G}(-d)\right)$ and $(4 d-5) L-K_{S} \in \mathrm{NS}(S)$. This is possible because

$$
s_{2}\left(N_{S / G}(-d)\right)=c+Q\left(L, K_{S}\right)
$$

where $Q$ is a degree 2 polynomial with integer coefficients. Then we apply formula (70), and we conclude that $M$ divides $z_{S}-\mu c_{2}(S)-\nu c_{1}(S)^{2}$ in $\mathrm{CH}_{0}(S)=\mathbb{Z}$. As $M$ is arbitrarily large, it follows that $z_{S}=\mu c_{2}(S)+\nu c_{1}(S)^{2}$.

\section{References}

[1] A. Beauville. Sur l'anneau de Chow d'une variété abélienne. Math. Ann. 273 (1986), no. $4,647-651$.

[2] A. Beauville, C. Voisin. On the Chow ring of a K3 surface, J. Algebraic Geom. 13 (2004), no. 3, 417-426.

[3] S. Bloch, V. Srinivas. Remarks on correspondences and algebraic cycles, Amer. J. of Math. 105 (1983) 1235-1253.

[4] M. de Cataldo, J. Migliorini. The Chow groups and the motive of the Hilbert scheme of points on a surface. J. Algebra 251 (2002), no. 2, 824-848.

[5] E. Colombo, B. Van Geemen. Note on curves in a Jacobian. Compositio Math. 88 (1993), no. 3, 333-353.

[6] G. Ellingsrud, L. Göttsche, M. Lehn. On the cobordism class of the Hilbert scheme of a surface. Journal of Algebraic Geometry, 10 (2001), 81-100.

[7] N. Fakhruddin, Algebraic cycles on generic Abelian varieties, Compositio Math. 100 (1996), 101-119.

[8] T. Gaffney, R. Lazarsfeld. On the ramification of the branched coverings of $\mathbb{P}^{n}$, Invent. Math. 59, 53-88 (1980).

[9] B. Gross, C. Schoen. The modified diagonal cycle on the triple product of a pointed curve. Ann. Inst. Fourier (Grenoble) 45 (1995), no. 3, 649-679.

[10] S.-I. Kimura. Chow groups are finite dimensional, in some sense. Math. Ann. 331 (2005), no. $1,173-201$.

[11] B. Moonen, Q. Yin. Some remarks on modified diagonals, arXiv:1405.1646, to appear in Communications in Contemporary Mathematics.

[12] K. O'Grady. Computations with modified diagonals, Atti Accad. Naz. Lincei Cl. Sci. Fis. Mat. Natur. Rend. Lincei (9) Mat. Appl. 25 (2014), no. 3, 249-274. 
[13] M. Shen, Ch. Vial. The Fourier transform for certain hyperkaehler fourfolds, to appear in Memoirs of the AMS.

[14] V. Voevodsky. A nilpotence theorem for cycles algebraically equivalent to zero. Internat. Math. Res. Notices, no. 4, 187-198, (1995).

[15] C. Voisin. Remarks on zero-cycles of self-products of varieties, in Moduli of vector bundles (Proceedings of the Taniguchi congress on vector bundles), Maruyama Ed., Decker (1994) 265-285.

[16] C. Voisin. On the Chow ring of certain algebraic hyper-Kähler manifolds, Pure and Applied Mathematics Quarterly, Volume 4, Number 3, (Special issue in honor of Fedya Bogomolov), 2008.

[17] C. Voisin. Infinitesimal invariants for cycles modulo algebraic equivalence and 1-cycles on Jacobians, Algebraic Geometry 2 (2014) 140-165.

[18] C. Voisin. Chow rings, decomposition of the diagonal and the topology of families, Annals of Math. Studies 187, Princeton University Press 2014.

[19] C. Voisin. Universally defined cycles, in preparation.

[20] Q. Yin. Finite dimensionality and cycles on powers of $K 3$ surfaces, arXiv:1404.0171.

Institut de Mathématiques de Jussieu

4 place Jussieu

Case 247

75252 Paris Cedex 05

France

claire.voisin@imj-prg.fr 